\title{
Harmonic spinors and metrics of positive curvature via the Gromoll filtration and Toda brackets
}

\author{
Diarmuid Crowley* \\ School of Mathematics and Statistics, \\ University of Melbourne, Australia \\ Thomas Schick ${ }^{\dagger}$ \\ Mathematisches Institut, \\ Universität Göttingen, Germany \\ Wolfgang Steimle \\ Institut für Mathematik, Universität Augsburg, Germany
}

August 30, 2018

\begin{abstract}
We construct non-trivial elements of order 2 in the homotopy groups $\pi_{8 j+1+*} \operatorname{Diff}\left(D^{6}, \partial\right)$ for $* \equiv 1,2(\bmod 8)$, which are detected through the chain

$$
\pi_{8 j+1+*} \operatorname{Diff}\left(D^{6}, \partial\right) \rightarrow \pi_{0} \operatorname{Diff}\left(D^{8 j+*+7}, \partial\right) \rightarrow K O_{*}=\mathbb{Z} / 2
$$

of the "assembling homomorphism" (giving rise to the Gromoll filtration) and the alpha-invariant.

These elements are constructed by means of Morlet's homotopy equivalence $\operatorname{Diff}\left(D^{6}, \partial\right) \simeq \Omega^{7}\left(P L_{6} / O_{6}\right)$ and Toda brackets in $P L_{6} / O_{6}$. We also construct non-trivial elements of order 2 in $\pi_{*} P L_{m}$ for every $m \geq 6$ and $* \equiv 1,2(\bmod 8)$ which are detected by the alpha-invariant.

As consequences, we (a) obtain non-trivial elements of order 2 in $\pi_{*} \operatorname{Diff}\left(D^{m}, \partial\right)$ for $m \geq 6$ such that $*+m \equiv 0,1(\bmod 8)$; (b) these elements remain non-trivial in $\pi_{*} \operatorname{Diff}(M)$ where $M$ is a closed spin manifold of the same dimension $m$ and $*>0$; (c) they act non-trivially on the corresponding homotopy group of the space of metrics of positive scalar curvature of such an $M$; in particular these homotopy groups are all nontrivial. The same applies to all other diffeomorphism invariant metrics of positive curvature, like the space of metrics of positive sectional curvature, or the space of metrics of positive Ricci curvature, provided they are non-empty.
\end{abstract}

*e-mail: dcrowley@unimelb.edu.au www: http://www.dcrowley.net/

†e-mail: thomas.schick@math.uni-goettingen.de www: http://www.uni-math.gwdg.de/schick

${ }^{\ddagger}$ e-mail: wolfgang.steimle@math.uni-augsburg.de www: http://www.math.uni-augsburg.de/prof/diff/arbeitsgruppe/steimle 
Further consequences are: (d) any closed spin manifold of dimension $m \geq 6$ admits a metric with harmonic spinors; (e) there is no analogue of the odd-primary splitting of $(P L / O)_{(p)}$ for the prime 2 ; (f) for any $b P_{8 j+4}$-sphere $(j \geq 1)$ of order which divides 4 , the corresponding element in $\pi_{0} \operatorname{Diff}\left(D^{8 j+2}, \partial\right)$ lifts to $\pi_{8 j-4} \operatorname{Diff}\left(D^{6}, \partial\right)$, i.e. lies correspondingly deep down in the Gromoll filtration.

\section{Introduction}

We use the Gromoll filtration [13] of $\Gamma^{n+1}=\pi_{0}\left(\operatorname{Diff}\left(D^{n}, \partial\right)\right)$ to study the topology of spaces of metrics of positive curvature and the topology of diffeomorphism groups for closed spin manifolds.

This Gromoll filtration $\cdots \subset \Gamma_{(k)}^{n+1} \subset \Gamma_{(k+1)}^{n+1} \subset \cdots \subset \Gamma_{(n)}^{n+1}=\Gamma^{n+1}$ is defined using the homomorphisms

$$
\lambda: \pi_{n-k}\left(\operatorname{Diff}\left(D^{k}, \partial\right)\right) \rightarrow \pi_{0}\left(\operatorname{Diff}\left(D^{n}, \partial\right)\right)=\Gamma^{n+1}
$$

simply by setting

$$
\Gamma_{(k)}^{n+1}:=\operatorname{Im}(\lambda) \subset \Gamma^{n+1} .
$$

Here $\lambda$ interprets a smooth family of diffeomorphisms of $D^{k}$ parametrized by $D^{n-k}$ as one diffeomorphism of $D^{n}$ (which preserves the first $n-k$ coordinates). Our notation is somewhat non-standard, $\Gamma_{(k)}^{n+1}$ is supposed to reflect the $k$ dimensional "disk of origin", as this is the relevant parameter for our applications. (The more traditional notation for what we call $\Gamma_{(k)}^{n+1}$ is $\Gamma_{n-k-1}^{n+1}$.)

Our main result is that certain important classes in $\Gamma^{n+1}$ have lifts all the way to $\Gamma_{(6)}^{n+1}$. "Important" here refers in particular to classes which have nontrivial $\alpha$-invariant, defined as follows and coinciding 11 with Adams' invariant $d_{\mathbb{R}}$ of [1, Section 7]. We consider the $\alpha$-invariant as a homomorphism to real $K$-homology (of a point)

$$
\alpha_{\Gamma}: \Gamma^{n+1} \rightarrow K O_{n+1},
$$

which factors in the following way

$$
\alpha_{\Gamma}: \Gamma^{n+1} \stackrel{\cong}{\stackrel{\cong}{\longrightarrow}} \Theta_{n+1} \longrightarrow \Omega_{n+1}^{\text {Spin }} \stackrel{\alpha_{\text {Spin }}}{\longrightarrow} K O_{n+1} .
$$

Here, $\Theta_{n+1}$ is the group of oriented diffeomorphism classes of homotopy spheres, and the isomorphism $\Sigma$ produces an exotic $(n+1)$-sphere from a diffeomorphism in $\Gamma^{n+1}$ by extending the latter by the identity map to $S^{n}$ and then clutching two $(n+1)$-disks using this diffeomorphism of $S^{n}$. The map to $\Omega_{n+1}^{\text {Spin }}$ assigns to a homotopy sphere the spin bordism class it represents (having a unique spin structure). Finally, the transformation $\alpha_{\text {Spin }}$ is the so-called Atiyah orientation; it assigns to a spin manifold the KO-valued index of its Dirac operator.

We will use many different versions of " $\alpha$-invariant" homomorphisms, defined on different spaces. In most cases, we will not distinguish them in notation but rather just write $\alpha$, the precise setting will be clear from the context.

Recall that $\Gamma^{n+1}$ is a finite abelian group for each $n$, and $K O_{n+1}=\mathbb{Z} / 2 \mathbb{Z}$ if $n \equiv 0,1$ modulo 8 , but is zero or infinite cyclic for all other degrees. Therefore,

\footnotetext{
${ }^{1}$ as proved in 25] Section 3]
} 
$\alpha_{\Gamma}$ is only interesting for $n \equiv 0,1(\bmod 8)$. It is a well known result of Adams [1. Section 7 and 12] that $\alpha$ is a split epimorphism in these cases (if $n>0$ ). Our main result improves this by constructing some elements with non-trivial $\alpha$-invariant deep in the Gromoll filtration:

1.1 Theorem. For all $j \geq 1$ and $\epsilon \in\{1,2\}$, there is a homotopy $(8 j+\epsilon)$-sphere with disk of origin not bigger than 6 and non-trivial $\alpha$-invariant, which is of order two in the group of homotopy spheres. In fact, somewhat more is true, namely

$$
\alpha: \pi_{8 j-7+\epsilon}\left(\operatorname{Diff}\left(D^{6}, \partial\right)\right) \rightarrow \Gamma_{(6)}^{8 j+\epsilon} \rightarrow K O_{8 j+\epsilon}
$$

is split surjective.

In [10] it was proven that $\alpha\left(\Gamma_{(7)}^{8 j+2}\right)=K O_{8 j+2}$. In this paper we improve this result in two ways: we reduce the disk of origin by one to $D^{6}$, and we also cover the dimensions $8 j+1$.

To our knowledge, lifts this far in the Gromoll filtration have rarely been constructed before. In addition, our construction methods seems to be novel. In [10], the first two authors constructed the required elements in $\Gamma_{(7)}^{8 j+2}$ as products between elements in $\pi_{\beta}\left(\operatorname{Diff}\left(D^{k}, \partial\right)\right)$ and $\pi_{\alpha}\left(S^{\beta}\right)$, a strategy which had been employed previously by Antonelli, Burghelea, and Kahn [4] and Burghelea and Lashoff [9].

In the present paper, we use a secondary product construction, more precisely, Toda brackets. In this way we implement the suggestion made in [10, Remark 2.15]. As a further application of the method, we prove Theorem 1.2 below. Let $\Gamma_{b P}^{4 i-1}:=\Sigma^{-1}\left(b P_{4 i}\right)$ be the subgroup of $\Gamma^{4 i-1}$ corresponding to those homotopy spheres which bound parallelizable manifolds. Since $b P_{4 i}$ is finite cyclic [17, $\Gamma_{b P}^{4 i-1} \cong b P_{4 i}$ has a unique subgroup of order 4 , which we denote by ${ }_{4} \Gamma_{b P}^{4 i-1}$.

1.2 Theorem. For all $j \geq 1$, every element of ${ }_{4} \Gamma_{b P}^{8 j+3}$ lies in $\Gamma_{(6)}^{8 j+3}$.

For a summary of earlier results on the Gromoll filtration of $b P_{4 k}$-homotopy spheres, see the bottom of the table in the Appendix A.

\subsection{Harmonic spinors and diffeomorphism groups}

It is an old question whether a given closed spin manifold $M$ admits harmonic spinors. Note that this depends on the Riemannian metric $M$, the more precise question therefore is whether $M$ admits a Riemannian metric such that its Dirac operator has non-trivial kernel.

This question has a long history. The many positive results all use the following strategy: if every metric admits a harmonic spinor, we are of course done. Otherwise, we look at the complement:

1.3 Definition. Define $\mathcal{R}^{\text {inv }}(M)$ to be the space of Riemannian metrics on $M$ with invertible Dirac operator.

It then suffices to show that this space is not contractible, so that it can not be equal to the (contractible) space of all Riemannian metrics.

Nigel Hitchin [15, Theorem 4.5] was the first to use essentially this method to prove that there are metrics with non-trivial harmonic spinor whenever $\operatorname{dim}(M) \equiv-1,0,1(\bmod 8)$. Later, Christian Bär 5 showed that the space 
of metrics with non-invertible Dirac operator on any spin manifold of dimension $m \equiv 3(\bmod 4)$ is non-empty. Waterstraat 34 showed that its components can be distinguished using the spectral flow of the Dirac operator, which actually is a relative index.

More specifically, we assume that there is a metric $g_{0} \in \mathcal{R}^{\text {inv }}(M)$. Choose an embedding of $D^{n}$ into $M$ and define $j: \operatorname{Diff}\left(D^{n}, \partial\right) \rightarrow \operatorname{Diff}(M)$ via extension of a diffeomorphism outside this embedded disk by the identity. We have the action map

$$
\operatorname{Diff}(M) \rightarrow \mathcal{R}^{\operatorname{inv}}(M) ; \quad f \mapsto f^{*} g_{0},
$$

given by pulling back $g_{0}$ by the diffeomorphism, which we may compose with the extension map $j$.

Our goal now is to use this sequence of maps to obtain non-trivial elements in $\pi_{n-m}\left(\mathcal{R}^{\text {inv }}(M), g_{0}\right)$. Indeed, we can use a relative index of the Dirac operator (the index difference to $g_{0}$ in the sense of Ebert [1])

$$
\text { ind-diff : } \pi_{n-m}\left(\mathcal{R}^{\text {inv }}(M), g_{0}\right) \rightarrow K O_{n+1} \text {. }
$$

Strictly speaking, in 11 the map is defined on the space of metrics of positive scalar curvature. However, the analytic condition required to construct it is not positive scalar curvature but merily the invertibility of the Dirac operator so that 11 literally applies.

The composition

$$
\begin{aligned}
& \pi_{n-m}\left(\operatorname{Diff}\left(D^{m}, \partial\right)\right) \rightarrow \pi_{n-m}(\operatorname{Diff}(M)) \\
& \quad \rightarrow \pi_{n-m}\left(\mathcal{R}^{\text {inv }}(M), g_{0}\right) \stackrel{\text { ind-diff }}{\longrightarrow} K O_{n+1}
\end{aligned}
$$

was introduced and studied by Hitchin [15. He proved that it is equal to the $\alpha$-invariant homomorphism.

With Theorem 1.1 above we produce the required input for Hitchin's method to work in almost all dimensions, therefore answering the question almost completely:

1.4 Theorem. Let $M$ be a closed spin manifold of dimension $m \geq 6$. Then $M$ admits a Riemannian metric with a non-trivial harmonic spinor. Indeed for each Riemannian metric $g_{0}$ in the complementary space $\mathcal{R}^{\operatorname{inv}}(M)$, the homotopy groups $\pi_{n-m}\left(\mathcal{R}^{\mathrm{inv}}(M), g_{0}\right)$ are non-trivial for $n \geq m$ and $n \equiv 0,1(\bmod 8)$.

Note that here $\mathcal{R}^{\operatorname{inv}}(M)$ is allowed to be empty, in which case the second statement is vacuous.

Proof. We start by proving the second assertion. The non-trivial classes of order 2 in $\pi_{n-m}\left(\operatorname{Diff}\left(D^{m}, \partial\right)\right)$ of Theorem 1.1 which are detected by $\alpha$, map to classes in $\pi_{n-m}\left(\mathcal{R}^{\text {inv }}(M), g_{0}\right)$ through the action homomorphism; the latter group is placed in the middle of the sequence (2), so the classes constructed in this way are non-trivial.

It follows that $\mathcal{R}^{\text {inv }}(M)$ is non-contractible (maybe empty) and therefore must be a strict subset of the contractible space of all Riemannian metrics on $M$, and the first assertion follows.

1.5 Remark. Bernd Ammann informs us that Theorem 1.4 also follows as a special case of work he carried out independently and in parallel together with Bunke, Pilca, and Nowaczyk. This work has not appeared yet in preprint form. 
When $\mathcal{R}^{\text {inv }}(M) \neq \emptyset$ our proof gives a bit more than stated in Theorem 1.4

1.6 Corollary. Under the assumptions of Theorem 1.4. and if $\mathcal{R}^{\mathrm{inv}}(M) \neq \emptyset$,

$$
\pi_{n-m}(\operatorname{Diff}(M), \mathrm{id}) \rightarrow K O_{n+1} \quad \text { and } \quad \pi_{n-m}\left(\mathcal{R}^{\mathrm{inv}}(M), g_{0}\right) \rightarrow K O_{n+1}
$$

are split epimorphisms for all $g_{0} \in \mathcal{R}^{\mathrm{inv}}(M)$. This provides infinitely many degrees where the homotopy groups contain a summand isomorphic to $\mathbb{Z} / 2$.

1.7 Remark. Note that $\mathcal{R}^{\text {inv }}(M)$ is non-empty if and only if the necessary condition for this is satisfied, namely that $\alpha(M)=0 \in K O_{m}$, compare [2].

1.8 Remark. In the situation of Corollary 1.6, suppose that the hypothesis $\mathcal{R}^{\text {inv }}(M) \neq \emptyset$ is omitted. Then our method still shows the existence of a split surjection $\pi_{n-m} \operatorname{Diff}(M) \rightarrow K O_{n+1}$, under the stronger hypothesis $n \geq m+2$, or after replacing $\operatorname{Diff}(M)$ by the "spin diffeomorphism group" whose elements are diffeomorphisms together with a lift of the derivative to the spin principal bundle. In this case the map to $K O$-theory is given by the $\alpha$-invariant of the mapping torus.

\subsection{Positive curvature}

An important application of Theorem 1.1 concerns the topology of spaces $\mathcal{R}_{\mathrm{c}}^{+}(M)$ of metrics of suitable positive curvature on a closed spin manifold $M$ of dimension $m$. Here, $\mathcal{R}_{\mathrm{c}}^{+}(M)$ can stand for any non-empty diffeomorphism invariant space of Riemannian metrics which is contained in $\mathcal{R}^{\text {inv }}(M)$. By the Schrödinger-Lichnerowicz formula this is the case for the space $\mathcal{R}_{s c}^{+}(M)$ of metrics of positive scalar curvature on $M$. We list the most studied examples of $\mathcal{R}_{\mathrm{C}}^{+}(M)$ :

- the space $\mathcal{R}_{s c}^{+}(M)$ of positive scalar curvature metrics,

- the space $\mathcal{R}_{R i c}^{+}$of positive Ricci curvature metrics,

- the space $\mathcal{R}_{\text {sec }}^{+}$of positive sectional curvature metrics,

- the space of $k$-positive Ricci curvature metrics for any $1 \leq k \leq \operatorname{dim}(M)$, interpolating between the first two cases.

We are studying the case where the corresponding space $\mathcal{R}_{\mathrm{c}}^{+}(M)$ is nonempty. The Schrödinger-Lichnerowicz formula entails that the first obstruction to the existence of a positive scalar curvature metric on $M$ is the index of the Dirac operator defined by its spin structure, i.e., $\alpha_{\text {Spin }}([M])$ of (10). When $M$ is simply connected of dimension $\geq 5$, Stolz 32 proved that $\mathcal{R}_{\mathrm{c}}^{+}(M) \neq \emptyset$ if and only if $\alpha(M)=0$. In general, the question of whether $\mathcal{R}_{\mathrm{c}}^{+}(X) \neq \emptyset$ is a deep problem which remains open, see [28, 30, 31.

We start at the other end and we assume that there is $g_{0} \in \mathcal{R}_{\mathrm{c}}^{+}(M)$, with $\mathcal{R}_{\mathrm{c}}^{+}(M)$ as above. As above, we have the embedding $j: \operatorname{Diff}\left(D^{m}, \partial\right) \rightarrow \operatorname{Diff}(M)$ and the action map

$$
\operatorname{Diff}(M) \rightarrow \mathcal{R}_{\mathrm{c}}^{+}(M) ; \quad f \mapsto f^{*} g_{0}
$$

Note that the map $\operatorname{Diff}(M) \rightarrow \mathcal{R}^{\text {inv }}(M)$ of Section 1.1 factors through this action map by the assumption $\mathcal{R}_{\mathrm{c}}^{+}(M) \subset \mathcal{R}^{\mathrm{inv}}(M)$. Corollary 1.6 therefore gives immediately the following corollary. 
1.9 Corollary. Let $M$ be a closed spin manifold of dimension $m \geq 6$ with a Riemannian metric $g_{0} \in \mathcal{R}_{\mathrm{c}}^{+}(M)$ for a space of metrics $\mathcal{R}_{\mathrm{c}}^{+}(M)$ as above. If $n \equiv 0,1(\bmod 8)$ and $n \geq m$, then $K O_{n+1}=\mathbb{Z} / 2$ and the composition

$$
\begin{aligned}
\pi_{n-m}\left(\operatorname{Diff}\left(D^{k}, \partial\right), \mathrm{id}\right) & \rightarrow \pi_{n-m}(\operatorname{Diff}(M), \mathrm{id}) \\
\rightarrow & \pi_{n-m}\left(\mathcal{R}_{\mathrm{c}}^{+}(M), g_{0}\right) \rightarrow \pi_{n-m}\left(\mathcal{R}^{\mathrm{inv}}(M), g_{0}\right) \rightarrow K O_{n+1}
\end{aligned}
$$

is a split epimorphism. In particular, also $\pi_{n-m}\left(\mathcal{R}_{\mathrm{c}}^{+}(M), g_{0}\right) \rightarrow K O_{n+1}=\mathbb{Z} / 2$ is a split epimorphism and $\mathcal{R}_{\mathrm{c}}^{+}\left(M, g_{0}\right)$ has infinitely many non-trivial homotopy groups.

1.10 Remark. Hitchin introduced precisely this method, applied to the space of metrics of positive scalar curvature in [15. However, at the time it was only known that

$$
\alpha: \pi_{k}\left(\operatorname{Diff}\left(D^{m}, \partial\right)\right) \rightarrow K O_{m+k+1}
$$

is surjective for $k=0$ or $k=1$, and $m+k \equiv 0,1(\bmod 8), m \geq 8$. Therefore, Hitchin with this method only could obtain information about $\pi_{0}\left(\mathcal{R}_{s c}^{+}(M)\right)$ and $\pi_{1}\left(\mathcal{R}_{s c}^{+}(M)\right)$.

Botvinnik, Ebert and Randal-Williams in the breakthrough paper [6] study the space of metrics of positive scalar curvature $\mathcal{R}_{s c}^{+}(M)$. They show that ind-diff : $\pi_{n-m}\left(\mathcal{R}_{s c}^{+}(M), g_{0}\right) \rightarrow K O_{n+1}$ is an epimorphism if $n \equiv 0,1(\bmod 8)$ and has infinite image if $K O_{n+1} \cong \mathbb{Z}$, i.e. $n \equiv 3(\bmod 4)$. Their methods are rather different from ours, in particular the family of metrics they obtain are very inexplicit and rely on surgery.

Hitchin's method, on the other hand, gives rather explicit families of metrics - at least if the family of diffeomorphisms used in the construction is explicit. We view this as one of the appealing features of our construction. Moreover, our method applies not only to scalar curvature, but to all metrics of positive curvature as listed above.

Note that in Hitchin's and therefore our construction of homotopy classes of metrics of positive scalar curvature, the corresponding families of metrics are obtained by pulling back $g_{0}$ with an appropriate family of diffeomorphisms which is supported on a small disk in $M$. This means that we only make a local change of the given initial metric $g_{0}$. We note that by the very way they are constructed these classes become trivial when mapped to the moduli space of metrics (in contrast to some elements of $\pi_{*}\left(\mathcal{R}_{s c}^{+}(M)\right)$ obtained in very different ways in [6, 14]).

\subsection{Toda brackets}

We now describe in more detail our method to prove the main Theorem[1.1] lifting certain exotic spheres deep in the Gromoll filtration, and additional results around this.

The starting point of the construction is a homotopy equivalence

$$
M: \operatorname{Diff}\left(D^{n}, \partial\right) \rightarrow \Omega^{n+1}\left(P L_{n} / O_{n}\right)
$$

due to Morlet [26], with a detailed proof by Burghelea and Lashof in [9, Theorem 4.4]. Recall that $P L_{n}$ is the simplicial group of piecewise linear homeomorphisms of $\mathbb{R}^{n}$ fixing the origin, with homotopy theoretic subgroup inclusion $O_{n} \rightarrow P L_{n}$ for the orthogonal group $O_{n}$. One sets $O:=\lim _{n \rightarrow \infty} O_{n}$, 
$P L:=\lim _{n \rightarrow \infty} P L_{n}$, and $P L / O:=\lim _{n \rightarrow \infty}\left(P L_{n} / O_{n}\right)$. There are of course stabilization maps $P L_{n} / O_{n} \rightarrow P L_{n+1} / O_{n+1} \rightarrow P L / O$ (we call all these stabilization maps $S$ ). We will also use the orientation preserving versions, denoted $S P L_{n}$, etc.

As checked in [9, Theorem 1.3] and [10, Lemma 2.5], under the isomorphism induced by $M$, the stabilization $\lambda$ defining the Gromoll filtration becomes the stabilization $S$, i.e. we have a commutative diagram

$$
\begin{array}{cccc}
\pi_{n-k}\left(\operatorname{Diff}\left(D^{k}, \partial\right)\right) \stackrel{\lambda}{\longrightarrow} \pi_{n-k-1}\left(\operatorname{Diff}\left(D^{k+1}, \partial\right)\right) \stackrel{\lambda}{\longrightarrow} \pi_{0}\left(\operatorname{Diff}\left(D^{n}, \partial\right)\right) \\
\cong \downarrow M_{*} & \cong M_{*} & \cong \downarrow M_{*} \\
\pi_{n+1}\left(P L_{k} / O_{k}\right) & \stackrel{S_{*}}{\longrightarrow} \pi_{n+1}\left(P L_{k+1} / O_{k+1}\right) & \stackrel{S_{*}}{\longrightarrow} \pi_{n+1}\left(P L_{n} / O_{n}\right),
\end{array}
$$

where the group in the bottom right corner is already stable, i.e. the stabilization map to $S_{*}: \pi_{n+1}\left(P L_{n} / O_{n}\right) \rightarrow \pi_{n+1}(P L / O)$ is an isomorphism [9, Theorem 4.6]. Indeed, as verified in [10, $\S 2]$, the fundamental theorem of smoothing theory [19. Theorem 7.3] gives an isomorphism

$$
\Psi: \Theta_{n+1} \cong \pi_{n+1}(P L / O)
$$

such that $S_{*} \circ M_{*}=\Psi \circ \Sigma: \Gamma^{n+1} \cong \pi_{n+1}(P L / O)$.

It follows that finding elements deep in the Gromoll filtration corresponds to lifting elements of $\pi_{n+1}(P L / O)$ to $\pi_{n+1}\left(P L_{k} / O_{k}\right)$. In the predecessor paper [10] this was achieved by using compositions

$$
\pi_{i}\left(S^{j}\right) \times \pi_{j}\left(P L_{k} / O_{k}\right) \rightarrow \pi_{i}\left(P L_{k} / O_{k}\right) .
$$

In this paper we show how Toda brackets on elements of $\pi_{*}\left(P L_{k} / O_{k}\right)$ can be used to go even deeper in the Gromoll filtration. Recall that the Toda bracket $\langle f, g, h\rangle$ of three homotopy classes of maps $f: X \rightarrow Y, g: Y \rightarrow Z, h: Z \rightarrow W$ is defined (as a secondary composition product) whenever the compositions of two consecutive maps are homotopic to constant maps. The Toda bracket is a set of homotopy classes of maps from $\Sigma X$ to $W$. The indeterminacy depends on the null homotopies one can choose.

To prove Theorem 1.1, we start with the unique element of order two,

$$
a_{P L_{6} / O_{6}} \in \pi_{7}\left(P L_{6} / O_{6}\right) \cong \Theta_{7} \cong \mathbb{Z} / 28
$$

where the first isomorphism is $\Psi_{*}^{-1} \circ S_{*}$ and the second isomorphism is found in [17. For $\epsilon \in\{1,2\}$, we now form Toda brackets

$$
\left\langle\mu_{8 j-8+\epsilon, 7}, 2_{7}, a_{P L_{6} / O_{6}}\right\rangle \subset \pi_{8 j+\epsilon}\left(P L_{6} / O_{6}\right)
$$

with certain elements

$$
\mu_{8 j-8+\epsilon, 7} \in \pi_{8 j-1+\epsilon}\left(S^{7}\right) \quad(j \geq 1)
$$

of order 2. These elements were constructed in [10, Section 2.3] and stabilize to a family of elements in $\pi_{8 j-8+\epsilon}^{s}$ constructed by Adams [1].

The main task is the to compute the $\alpha$-invariant of an element in this Toda bracket. As an ingredient, we use the fact that the $\alpha$-invariant from (10) is induced by a map of spaces

$$
\alpha_{P L / O}: P L / O \rightarrow \Omega^{\infty} \mathbf{K O}
$$


so that it is natural with respect to composition products and Toda brackets. We obtain:

1.11 Theorem. (a) For all $j \geq 1$ and $\epsilon \in\{1,2\}$, any element in the Toda bracket

$$
\left\langle\mu_{8 j-8+\epsilon, 7}, 2_{7}, a_{P L_{6} / O_{6}}\right\rangle \subset \pi_{8 j+\epsilon}\left(P L_{6} / O_{6}\right)
$$

has non-trivial $\alpha$-invariant.

(b) There is an element of order 2 in this Toda bracket.

Proof of Theorem 1.1. The theorem follows from Theorem 1.11 by translating the elements from (b) back through the Morlet equivalence of (1.3). Explicitly, let $b \in \pi_{8 j+\epsilon}\left(P L_{6} / O_{6}\right)$ be an element of order 2 as in Theorem 1.11(a). Via the Morlet isomorphism, we obtain $M_{*}^{-1}(b) \in \pi_{8 j-7+\epsilon}\left(\operatorname{Diff}\left(D^{6}, \partial\right)\right)$ of order 2 with non-trivial $\alpha$-invariant and hence $\alpha: \pi_{8 j-7+\epsilon}\left(\operatorname{Diff}\left(D^{6}, \partial\right)\right) \rightarrow K O_{8 j+\epsilon}$ is split surjective.

\subsection{The space $P L / O$ and $\operatorname{Im}(J)$-homotopy spheres}

Recall $\pi_{k}^{s}:=\operatorname{colim}_{i \rightarrow \infty} \pi_{i+k}\left(S^{i}\right)$ and the $J$-homomorphism $J_{*}: \pi_{*}(O) \rightarrow \pi_{*}^{s}$. The Kervaire-Milnor exact sequence [17,

$$
0 \rightarrow b P_{n+2} \rightarrow \Theta_{n+1} \stackrel{\Phi}{\longrightarrow} \operatorname{coker}\left(J_{n+1}\right)
$$

is an exact sequence of finite abelian groups which is split short exact at any odd prime $p$ by a result of Brumfiel [7, Theorem 1.3]. Actually, there is a canonical splitting of the $p$-localization

$$
(P L / O)_{(p)} \sim N_{(p)} \times C_{(p)}
$$

with isomorphisms $\pi_{*}\left(N_{(p)}\right) \cong\left(b P_{*+1}\right)_{(p)}$ and $\pi_{*}\left(C_{(p)}\right) \cong \operatorname{coker}\left(J_{*}\right)_{(p)}$ which, via the isomorphism $\Psi: \Theta_{*} \cong \pi_{*}(P L / O)$, induce Brumfiel's splitting of $\left(\Theta_{*}\right)_{(p)}$; see [23, Theorem 6.8 (iii)] and [24]2. Here we have written $A_{(p)}$ for the localization of an abelian group $A$. The following theorem shows that such a splitting cannot exist at the prime $p=2$.

1.12 Theorem. There is no space $N_{(2)}$ with map $t: N_{(2)} \rightarrow(P L / O)_{(2)}$ such that $t_{*}: \pi_{*}\left(N_{(2)}\right) \rightarrow \pi_{*}\left((P L / O)_{(2)}\right)$ is injective with image $\Psi^{-1}\left(\left(b P_{*+1}\right)_{(2)}\right)$.

Proof. The exotic sphere corresponding to the element $a_{P L_{6} / O_{6}} \in \pi_{7}\left(P L_{6} / O_{6}\right)$ from (3) is a $b P$-sphere. So it would define an element $t_{*}^{-1}\left(a_{N}\right) \in \pi_{7}\left(N_{(2)}\right)$ if such a space and map existed. Then, any element in the Toda bracket $\left\langle\eta_{7}, 2_{7}, t_{*}^{-1}\left(a_{N}\right)\right\rangle \subset \pi_{9}\left(N_{(2)}\right)$ would map under $\Psi \circ t_{*}$, perhaps up to odd multiples, a $b P$-sphere in dimension 9 . But any $b P$-sphere has trivial $\alpha$-invariant, contradicting Theorem 1.11 .

Let $G_{n}$ be the topological monoid of self-homotopy equivalences of the $(n-1)$-sphere:

$$
G_{n}:=\left\{\phi: S^{n-1} \stackrel{\simeq}{\longrightarrow} S^{n-1}\right\} ; \quad G=\lim _{n \rightarrow \infty} G_{n},
$$

\footnotetext{
${ }^{2}$ These results there are stated for the space $(T O P / O)_{(p)}$ but $(P L / O)_{(p)} \simeq(T O P / O)_{(p)}$
} at odd primes by Kirby-Siebenmann [18, V Theorem 5.3]. 
and let $S G_{n}$ and $S G$ be their orientation preserving variants, consisting of maps of degree 1 . To consider the situation when $p=2$ we recall that [23, $\mathrm{V} \S 4$ ] defined an equivalence

$$
\psi: S G \simeq \mathcal{J}_{\infty} \times C_{\infty},
$$

where $\mathcal{J}_{\infty}:=\prod_{p} \mathcal{J}_{p}$ for certain $p$-local spaces $\mathcal{J}_{p}, C_{\infty}:=\prod_{p} C_{p}$ and we have $\pi_{*}\left(\mathcal{J}_{\infty}\right) \cong \operatorname{Im}\left(J_{*}\right) \oplus \operatorname{Tors}\left(K O_{*}\right)$ and $\pi_{*}(C) \cong \operatorname{coker}\left(J_{*}\right) / \operatorname{Tors}\left(K O_{*}\right)$. In Section 4 we show that the splitting (6) gives rise to a splitting of the $\alpha$-invariant

$$
s_{*}: \operatorname{Tors}\left(K O_{n+1}\right) \rightarrow \operatorname{coker}\left(J_{n+1}\right) .
$$

Using the Kervaire-Milnor homomorphism $\Phi: \Theta_{n+1} \rightarrow \operatorname{coker}\left(J_{n+1}\right)$, we say that a homotopy $(n+1)$-sphere $\Sigma$ is an $\operatorname{Im}(J)$-sphere if

$$
\Phi(\Sigma) \in s_{*}\left(\operatorname{Tors}\left(K O_{n+1}\right)\right)
$$

and we define $\Theta_{n+1}^{J} \subset \Theta_{n+1}$ to be the subgroup of $\operatorname{Im}(J)$-homotopy spheres. We compute in Lemma 4.4

$$
\Theta_{n+1}^{J} \cong b P_{n+2} \oplus \operatorname{Tors}\left(K O_{n+1}\right)
$$

with $\alpha\left(b P_{n+1}\right)=\{0\}$ and $\alpha\left(\Theta_{n}^{J}\right)=\operatorname{Tors}\left(K O_{n}\right)$.

We show that Theorem 1.1] can be made more explicit, regarding $\operatorname{Im}(J)$ spheres. This relies on an $\operatorname{Im}(J)$-version of Theorem 1.11, which in turn uses $\operatorname{Im}(J)$-versions $P L_{n}^{J}$ of $P L_{n}$ etc.

1.13 Theorem. For all $j \geq 1$ and $\epsilon \in\{1,2\}$, there is an $\operatorname{Im}(J)$-homotopy sphere $\Sigma \in \Theta_{8 j+\epsilon}^{J}$ of order two with $\alpha(\Sigma)=1$ and disk origin at most 6 .

\subsection{Some new elements of $\pi_{*}\left(P L_{m}\right)$}

Above, our interest in the space $P L_{6} / O_{6}$ arose due to the Morlet equivalence $\operatorname{Diff}\left(D^{6}, \partial\right) \simeq \Omega^{7}\left(P L_{6} / O_{6}\right)$. But the space $P L_{6} / O_{6}$, and more generally the spaces $P L_{m} / O_{m}, P L_{m}$ and $T O P_{m}, T O P_{m} / O_{m}$, have an important role in smoothing theory and a long history of study in their own right. Here $T O P_{m}$ is the space base of base-point preserving homeomorphisms of $\mathbb{R}^{m}$ and $T O P_{m} / O_{m}$ is its quotient by the orthogonal group, with $T O P=\lim _{m} T O P_{m}$ and $T O P / O=\lim _{m} T O P_{m} / O_{m}$.

We mention just one recent major breakthrough concerning the homotopy theory of the spaces above, based on the fundamental work of Galatius and Randal-Williams [12]: Weiss [37, Appendix B] proves that the Pontrjagin class defines a non-trivial homomorphism

$$
\pi_{4 k-1}\left(T O P_{m}\right) \rightarrow \mathbb{Q}
$$

in a range of dimensions where the corresponding homomorphism on $\pi_{4 k-1}\left(O_{m}\right)$ vanishes. Hence Weiss shows the existence of classes in $\pi_{4 k-1}\left(T O P_{m}\right) \otimes \mathbb{Q}$ which map non-trivially to $\pi_{4 k-1}\left(T O P_{m} / O_{m}\right) \otimes \mathbb{Q}$.

Below we describe how computations with Toda brackets give new information about the 2-primary homotopy structure of the spaces listed above, and specifically about 2 -torsion in $\pi_{*}\left(P L_{m}\right)$ and $\pi_{*}\left(T O P_{m}\right)$. 
Consider the following homotopy commutative diagram which gives a space level description of the $\alpha$-invariant (see Section 2.3):

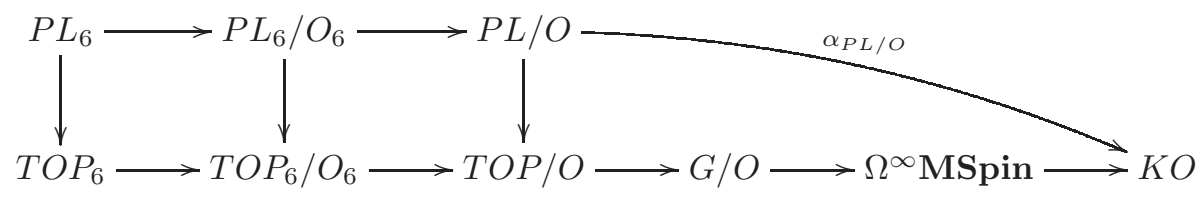

For any space $X$ in the above diagram we write $\alpha: \pi_{*}(X) \rightarrow K O_{*}$ for the map induced on homotopy groups by the corresponding map $X \rightarrow K O$.

The methods described in Section 1.3 required as input the order two homotopy class $a_{P L_{6} / O_{6}} \in 2 \pi_{7}\left(P L_{6} / O_{6}\right)$, the subgroup of elements divisible by 2. In Section 3.2 we show that $a_{P L_{6} / O_{6}}$ lifts to an element of order two $a_{P L_{6}} \in 2 \pi_{7}\left(P L_{6}\right)$ and this allows us to prove

1.14 Theorem. For all $j \geq 1, \epsilon \in\{1,2\}$ and $m \geq 6$, the $\alpha$-invariant

$$
\alpha: \pi_{8 j+\epsilon}\left(P L_{m}\right) \rightarrow K O_{8 j+\epsilon}
$$

is a split surjection. Hence the same holds for $\alpha: \pi_{8 j+\epsilon}\left(T O P_{m}\right) \rightarrow K O_{8 j+\epsilon}$, $\alpha: \pi_{8 j+\epsilon}\left(P L_{m} / O_{m}\right) \rightarrow K O_{8 j+\epsilon}$ and $\alpha: \pi_{8 j+\epsilon}\left(T O P_{m} / O_{m}\right) \rightarrow K O_{8 j+\epsilon}$.

The rest of this paper is organised as follows: In Section 2 we establish basic facts about Toda brackets in the space $S G_{n}$, mod 2 homotopy groups and the space level $\alpha$-invariant. Section 3 is about the $\alpha$-invariant on $P L_{6} / O_{6}$ and $P L_{6}$ and Theorems 1.11 and 1.14 are proven there. Section 4 covers $\operatorname{Im}(J)$-homotopy spheres and contains the proofs of Theorems 1.13 and 1.2 .

Acknowledgements: We would like to thank Peter May for helpful comments concerning the splitting of $(P L / O)_{(p)}$ in (5). We also thank the referree for many suggestions which have improved the presentation.

\section{Toda brackets, $\pi_{*}^{M}(X)$ and the $\alpha$-invariant}

\subsection{Toda brackets in $S G_{n}$}

In this subsection we review the canonical homomorphism

$$
I: \pi_{k}\left(S G_{n}\right) \rightarrow \pi_{k+n}\left(S^{n}\right)
$$

and Toda brackets.

Let $h: X \rightarrow S G_{n}$ be a map. The adjoint of $h$ is given by

$$
\widehat{h}: X \times S^{n-1} \rightarrow S^{n-1}, \quad(x, y) \mapsto h(x)(y) .
$$

For any map $\psi: X \times Y \rightarrow Z$, the Hopf construction on $\psi[38, \mathrm{Ch}$. XI, $\S 4]$ is the map

$$
S \psi: X * Y \rightarrow \Sigma Z, \quad[x, t, y] \mapsto[\psi(x, y), t],
$$

were $*$ denotes join and $\Sigma$ denotes suspension. For any space $X$ we identify $X * S^{n-1}=\Sigma^{n} X$. Identifying further $\Sigma^{n} S^{k}$ with $S^{n+k}$ we obtain a map

$$
I_{s p}: \operatorname{Map}\left(X, S G_{n}\right) \rightarrow \operatorname{Map}\left(\Sigma^{n} X, S^{n}\right), \quad h \mapsto S \widehat{h} .
$$


Passing to path-components we obtain a map

$$
I_{s p}:\left[X, S G_{n}\right] \rightarrow\left[\Sigma^{n} X, S^{n}\right], \quad[h] \mapsto[S \widehat{h}] .
$$

If we set $X=S^{k}$, we obtain the homomorphism

$$
I: \pi_{k}\left(S G_{n}\right) \rightarrow \pi_{k+n}\left(S^{n}\right)
$$

noting that we can identify unpointed with pointed homotopy classes, since $S G_{n}$ and $S^{1}$ are connected $H$-spaces and since $S^{n}$ is simply-connected for $n>1$. If $0<k<n-1$, then $\pi_{k}\left(S G_{n}\right) \cong \pi_{k}(S G)$ and $\pi_{k+n}\left(S^{n}\right) \cong \pi_{k}^{s}$ are stable and $I$ is an isomorphism

$$
I: \pi_{k}(S G) \stackrel{\cong}{\longrightarrow} \pi_{k}^{s}
$$

2.1 Remark. The isomorphism $I$ is often taken as an identification, e.g. in [10].

Recall that if

$$
X \stackrel{f}{\rightarrow} Y \stackrel{g}{\rightarrow} Z \stackrel{h}{\rightarrow} W
$$

is a sequence of continuous maps such that both composites $g f$ and $h g$ are nullhomotopic, then the Toda bracket

$$
\langle f, g, h\rangle \subset[\Sigma X, W]
$$

is defined as the set of all homotopy classes constructed in the following way: Choose nullhomotopies $H$ and $K$ of $g f$ and $h g$ so to obtain two nullhomotopies $h_{*} H$ and $f^{*} K$ of the triple composite $h g f$, thus a map from $\Sigma X$ to $W$. By construction, if $k: W \rightarrow W^{\prime}$ is another map, then

$$
k_{*}\langle f, g, h\rangle \subset\langle f, g, k h\rangle \subset\left[\Sigma X, W^{\prime}\right] .
$$

2.2 Lemma. Let $f: S^{i} \rightarrow S^{j}, g: S^{j} \rightarrow S^{k}$ and $h: S^{k} \rightarrow S G_{n}$ be maps such that $\langle f, g, h\rangle$ is defined. Then

$$
I(\langle f, g, h\rangle) \subset\left\langle\Sigma^{n} f, \Sigma^{n} g, I h\right\rangle \subset \pi_{i+1+n}\left(S^{n}\right) .
$$

Proof. Since $I_{s p}$ is natural in $X, I h$ is given by suspending $h$ first $n$ times and then postcomposing with

$$
\varepsilon:=I_{s p}\left(\operatorname{id}_{S G_{n}}\right): \Sigma^{n}\left(S G_{n}\right) \rightarrow S^{n} .
$$

Applying this reasoning to $\langle f, g, h\rangle$ instead of $h$ we have

$$
\begin{aligned}
& I(\langle f, g, h\rangle)=\varepsilon_{*}\left(\Sigma^{n}\langle f, g, h\rangle\right) \\
& \subset \varepsilon_{*}\left(\left\langle\Sigma^{n} f, \Sigma^{n} g, \Sigma^{n} h\right\rangle\right) \subset\left\langle\Sigma^{n} f, \Sigma^{n} g, \varepsilon \circ\left(\Sigma^{n} h\right)\right\rangle
\end{aligned}
$$

where the last map is $I h$.

\subsection{Mod 2 homotopy groups}

In this subsection we recall how maps of mod 2 Moore spaces are related to certain Toda brackets.

Let

$$
M_{k}:=S^{k} \cup_{2} e^{k+1}
$$


be the mod-2 Moore space, and $c: M_{k} \rightarrow S^{k+1}$ the map collapsing $S^{k}$ to a point. If $X$ is a simply-connected space and $x: S^{k} \rightarrow X$ is such that $2 x=0 \in \pi_{k}(X)$, then $x$ can be extended to a map $\bar{x}: M_{k} \rightarrow X$. Moreover, if $y: S^{i-1} \rightarrow S^{k}$ is a map such that $2 y=0 \in \pi_{i-1}\left(S^{k}\right)$ then there is a map $\overline{S y}: S^{i} \rightarrow M_{k}$ such that $c \circ \overline{S y}=S y$.

Thus we can form the composition $\bar{x} \circ \overline{S y}: S^{i} \rightarrow M_{k} \rightarrow X$, and it follows from the definitions that

$$
\bar{x} \circ \overline{S y} \in\langle y, 2, x\rangle \subset \pi_{i}(X) .
$$

Here we identify $\pi_{k}\left(S^{k}\right)=\mathbb{Z}$ via the mapping degree. Moreover, the choices in the construction of $\overline{S y}$ and $\bar{x}$ correspond precisely to the indeterminacy in the Toda bracket.

To apply the above, we will be interested in pointed homotopy classes of base-point preserving maps $\bar{x}: M_{k} \rightarrow X$. Let $X$ be a simply connected space and define the $k$-th homotopy set with $\mathbb{Z} / 2$-coefficients, see [27, §3], by

$$
\pi_{k}^{M}(X):=\left[M_{k}, X\right]_{*} .
$$

Note that 27] uses different notation, with $\pi_{k}^{M}(X)=\pi_{k+1}(X ; \mathbb{Z} / 2)$. Notice that for $k \geq 2, M_{k}$ is a suspension and so a co-H-space and so $\pi_{k}^{M}(X)$ has a natural group structure

The cofibration sequence $S^{k} \stackrel{i}{\rightarrow} M_{k} \stackrel{c}{\rightarrow} S^{k+1}$ comes with a long exact Puppe sequence [27, §18]

$$
\cdots \rightarrow \pi_{k+1}(X) \stackrel{\times 2}{\longrightarrow} \pi_{k+1}(X) \stackrel{c^{*}}{\longrightarrow} \pi_{k}^{M}(X) \stackrel{i^{*}}{\longrightarrow} \pi_{k}(X) \stackrel{\times 2}{\longrightarrow} \pi_{k}(X) \rightarrow \ldots .
$$

For an abelian group $A$, let ${ }_{2} A \subset A$ denote the subgroup of elements of order less than or equal to two and let $A / 2$ denote the quotient $A / 2 A$. The long exact sequence above gives rise to a short exact sequence, see [27, §3],

$$
0 \rightarrow \pi_{k+1}(X) / 2 \stackrel{c^{*}}{\rightarrow} \pi_{k}^{M}(X) \stackrel{i^{*}}{\rightarrow}{ }_{2} \pi_{k}(X) \rightarrow 0 .
$$

Denote by $\eta: S^{k+1} \rightarrow S^{k}$ the non-trivial homotopy class. The following lemma is probably well known to experts, but as we did not find a reference, we give a proof.

2.3 Lemma. Let $x \in{ }_{2} \pi_{k}(X)$ and let $\bar{x} \in \pi_{k}^{M}(X)$ have $i^{*}(\bar{x})=x$. Then $2 \bar{x}=[x \circ \eta] \in \pi_{k+1}(X) / 2$.

Proof. By naturality it is enough to consider the case where $X=M_{k}$ and $\bar{x}=1 \in \pi_{k}^{M}\left(M_{k}\right)$, represented by the identity map. Now $\pi_{k}\left(M_{k}\right)=\mathbb{Z} / 2[i]$ where $i: S^{k} \rightarrow M_{k}$ is the inclusion, and $\pi_{k+1}\left(M_{k}\right)=\mathbb{Z} / 2[i \circ \eta]$. Hence the short exact sequence (8) becomes

$$
0 \rightarrow \mathbb{Z} / 2 \stackrel{c^{*}}{\longrightarrow} \pi_{k}^{M}\left(M_{k}\right) \stackrel{i^{*}}{\longrightarrow} \mathbb{Z} / 2 \rightarrow 0,
$$

and we must show that $2 \in \pi_{k}^{M}\left(M_{k}\right)$ is equal to $c^{*}(i \circ \eta)$, i.e. that the sequence does not split, or equivalently that $\pi_{k}^{M}\left(M_{k}\right) \cong \mathbb{Z} / 4$. This is exactly the statement of [27, Corollary 7.3]. 


\subsection{The $\alpha$-invariant}

Recall that the $\alpha$-invariant is a morphism of (homotopy) ring spectra

$$
\alpha: \text { MSpin } \rightarrow \text { KO }
$$

inducing a ring homomorphism

$$
\alpha: \Omega_{*}^{\mathrm{Spin}} \rightarrow K O_{*}
$$

on homotopy groups. In this section we present the construction of a continuous map

$$
c: G / O \rightarrow \Omega^{\infty} \text { MSpin }
$$

and give some properties. The interest of this construction is that, when precomposed with the inclusion $P L / O \rightarrow G / O$ and postcomposed with the $\alpha$-invariant, we obtain a group homomorphism

$$
\alpha: \pi_{*}(P L / O) \rightarrow K O_{*}
$$

which we will use to detect non-triviality of certain exotic spheres. Similarly, we may compose with the projection $G \rightarrow G / O$ to obtain a different group homomorphism (still denoted by the same letter)

$$
\alpha: \pi_{*}^{s} \cong \pi_{*}(G) \rightarrow K O_{*}
$$

where we use the isomorphism $I: \pi_{*} G \cong \pi_{*}^{s}$ from subsection 2.1.

We start by recalling some facts about orientations. For a (homotopy) ring spectrum $\mathbf{R}$ and an $n$-dimensional spherical fibration $p$ over $B$, with Thom space $T(p)$, an $\mathbf{R}$-orientation of $p$ is a choice of Thom class $\tau \in \mathbf{R}^{n}(T(p)$,*). The group of units $G L_{1}\left(\mathbf{R}^{0}(B)\right)$ in the ring $\mathbf{R}^{0}(B)$ acts on the set of orientations by product and it is a consequence of the Thom isomorphism that this action is free and transitive. Thus if $\tau_{1}$ and $\tau_{2}$ are two $\mathbf{R}$-orientations of $p$, there is a difference class $\tau_{1} / \tau_{2} \in G L_{1}\left(\mathbf{R}^{0}(B)\right)$, defined uniquely by the property that

$$
\tau_{2} \cdot\left(\tau_{1} / \tau_{2}\right)=\tau_{1} \in \mathbf{R}^{n}(T(p), *) .
$$

As usual, let $G L_{1}(\mathbf{R}) \subset \Omega^{\infty} \mathbf{R}$ consist of those components which project to elements in $G L_{1}\left(\pi_{0} \mathbf{R}\right)$. With this notation the difference class $\tau_{2} / \tau_{1}$ (just as any element in $\left.G L_{1}\left(\mathbf{R}^{0}(B)\right)\right)$ is given by an unpointed homotopy class of maps $B \rightarrow G L_{1}(\mathbf{R})$. (The space $G L_{1}(\mathbf{S})$ is classically denoted by $F$ and the component of $G L_{1}(\mathbf{K O})$ containing the unit is classically denoted by $B O^{\otimes}$.)

In our setting $\mathbf{R}=\mathbf{M S p i n}$. The universal bundle over $G_{n} / \operatorname{Spin}_{n}$ (classified by the projection to $B \operatorname{Spin}_{n}$ ) has two canonical MSpin-orientations, $\tau_{1}$ (given by the spin structure) and $\tau_{2}$ (by the fiber homotopy trivialization). The difference classes $\tau_{1} / \tau_{2}$ for varying $n$ stabilize to yield a map

$$
c: G / \text { Spin } \rightarrow G L_{1}(\text { MSpin }) \subset \Omega^{\infty} \text { MSpin. }
$$

An explicit description of this map is as follows: As the universal bundle $p_{n}$ over $G_{n} / \operatorname{Spin}_{n}$ pulls back from the universal bundle over $B \operatorname{Spin}_{n}$, there is an induced map on Thom spaces $T\left(p_{n}\right) \rightarrow \mathbf{M S p i n}_{n}$. The fiber homotopy 
trivialization of $p_{n}$ induces a homotopy equivalence $T\left(p_{n}\right) \simeq \Sigma_{+}^{n}\left(G_{n} / \operatorname{Spin}_{n}\right)$, so we get a map $\Sigma_{+}^{n}\left(G_{n} / \operatorname{Spin}_{n}\right) \rightarrow \mathbf{M S p i n}_{n}$ adjoint to a map

$$
c: G_{n} / \operatorname{Spin}_{n} \rightarrow \Omega^{n} \mathbf{M S p i n}_{n} \subset \Omega^{\infty} \mathbf{M S p i n}
$$

of spaces. By construction, the base-point of $G_{n} / \operatorname{Spin}_{n}$ maps to the unit $1 \in$ $\pi_{0}$ MSpin; it follows that $c$ takes values in $G L_{1}$ (MSpin).

2.4 Remark. If GSpin $\rightarrow G$ denotes the 1-connected covering, then the composite

$$
G \text { Spin/Spin } \rightarrow G / \operatorname{Spin} \rightarrow G / O
$$

is a homotopy equivalence, hence a map on $G /$ Spin gives rise to a map on $G / O$.

The homotopy groups in positive degrees of $G L_{1}(\mathbf{K O})$ are canonically identified with those of $K O$ by means of the canonical homotopy equivalence (of spaces)

$$
G L_{1}(\mathbf{K O}) \simeq \Omega_{0}^{\infty} \mathbf{K O} \times\{ \pm 1\} .
$$

In the following, we denote the sphere spectrum by $\mathbf{S}$.

2.5 Lemma. The map $\alpha: \pi_{*}(P L / O) \rightarrow K O_{*}$ is compatible with precomposition: If $x \in \pi_{n}(P L / O)$ and $f: S^{n+k} \rightarrow S^{n}$ with $k>0$, then

$$
\alpha(x \circ f)=\alpha(x) \cdot S f=\alpha(x) \cdot \alpha(S f) \in K O_{n+k}
$$

where $S f \in \pi_{k}^{s}$ is the stabilization of $f$. In the middle term, multiplication by $f$ is through the unit map $\mathbf{S} \rightarrow \mathbf{K O}$ of the ring spectrum $\mathbf{K O}$ (given by $1 \in \pi_{0} \mathbf{K O}$ ); in the last term we view $S f$ as an element in $\pi_{k}(G)$ (through the isomorphism $I$ from above) which maps to an element in $\pi_{k}(G / \mathrm{Spin})$, to which the $\alpha$-invariant may be applied.

Proof. As $\alpha$ is continuous, it is compatible with precomposition, and it is wellknown that the action of $\pi_{*}^{s}$ by precomposition on the homotopy groups of a ring spectrum agrees with the one through the unit map. This proves the first identity.

Now recall that $G L_{1}(\mathbf{S}) \simeq G$. Under this equivalence, $G L_{1}$ of the unit map $\mathbf{S} \rightarrow$ MSpin factors through $c: G /$ Spin $\rightarrow G L_{1}$ (MSpin) via the canonical projection $G \rightarrow G /$ Spin; this follows from the explicit description of $c$ as given above. As $\alpha$ : MSpin $\rightarrow$ KO is a ring map, this implies the second equality.

Finally, we would like to identify the map $c$, on homotopy groups, with the canonical map from almost framed bordism to spin bordism. Recall that a homotopy class of (unpointed) maps $f: S^{n} \rightarrow G$ /Spin gives rise to a degree one normal map of a spin manifold $M_{f}^{n}$ onto the $n$-sphere, in particular to a spin bordism class $\left[M_{f}\right] \in \Omega_{n}^{\text {Spin }}$, represented by a pointed homotopy class $c_{f}: S^{n} \rightarrow \Omega^{\infty}$ MSpin.

On the other hand, the composite $c \circ f$ is an unpointed homotopy class $S^{n} \rightarrow \Omega^{\infty}$ MSpin. Since the short exact sequence

$$
0 \rightarrow \pi_{n} \text { MSpin } \rightarrow\left[S^{n}, \Omega^{\infty} \text { MSpin }\right] \rightarrow \pi_{0} \text { MSpin } \rightarrow 0
$$

is canonically split, there is a canonical projection

$$
\pi:\left[S^{n}, \Omega^{\infty} \text { MSpin }\right] \rightarrow \pi_{n} \text { MSpin. }
$$


2.6 Lemma. We have $c_{f}=\pi(c \circ f)$ as pointed homotopy classes.

(Since $c \circ f$ lands in the 1-component of MSpin, the pointed map $\pi(c \circ f)$ is represented by the loop space difference $c \circ f-1$.)

Proof. We first note that, for $f: S^{n} \rightarrow G_{k} / \operatorname{Spin}_{k}$, the map $c \circ f$ is adjoint to the composite

$$
S^{k} \wedge S_{+}^{n}=T\left(\varepsilon^{k}\right) \rightarrow T(\gamma) \rightarrow \operatorname{MSpin}_{k}
$$

where $\gamma$ is the pull-back of the universal bundle $p_{k}$ along $f$ and the first map comes from the fiber homotopy trivialization classified by $f$.

Now observe that the projection $\pi$ is characterized by the properties that it is the identity on the subgroup $\pi_{n}$ MSpin and that it sends constant maps to zero. One can verify by inspection that these properties also hold for the composite

$$
\left[S^{n}, \Omega^{k} \operatorname{MSpin}_{k}\right] \cong\left[S^{k} \wedge S_{+}^{n}, \operatorname{MSpin}_{k}\right]_{*} \rightarrow \pi_{n+k} \mathbf{M S p i n}_{k}
$$

where we pull back along along the Thom collapse $S^{n+k} \rightarrow S^{k} \wedge S_{+}^{n}$ of the $n$-sphere embedded trivially in $S^{n+k}$. Hence, the pointed class $\pi(c \circ f)$ is represented by pull-back of (9) along the Thom collapse. But this composite is just the definition of $c_{f}$.

\section{Toda brackets and homotopy spheres}

\subsection{The $\alpha$-invariant on $\pi_{*}\left(P L_{6} / O_{6}\right)$}

Recall that $\pi_{n+1}(P L / O)$ is identified, via smoothing theory, with the group of homotopy $(n+1)$-spheres. Denote by $a_{P L / O} \in \pi_{7}(P L / O) \cong \mathbb{Z} / 28$ the unique element of order 2. Also, for $j \geq 1$ and $\epsilon \in\{1,2\}$, let

$$
f: S^{8 j-1+\epsilon} \rightarrow S^{7}
$$

be any homotopy class such that

- $\alpha(f)=1$,

- $f$ is of order 2 , and

- $f$ is the suspension of some $f^{\prime} \in \pi_{8 j-2+\epsilon}\left(S^{6}\right)$, equally of order 2 .

In the case $\epsilon=1$, such elements $f$ exist for all $j \geq 1$ by [1, Theorem 1.2], where they are called $\mu_{8 j+1} \in \pi_{8 j+1}^{s}$. (Adams was mainly concerned about elements in the stable stems; in [10, Lemma 2.14] it was verified that the corresponding elements descend to order 2 elements on $S^{7}$, actually, on $S^{5}$.) We can precompose any such element $f$ by the non-trivial element $\eta \in \pi_{8 j+2}\left(S^{8 j+1}\right)$ to obtain a corresponding element for $\epsilon=2$, in view of Lemma 2.5 and the ring structure on $K O_{*}$.

As both $f$ and $a_{P L / O}$ have order 2 , the Toda bracket

$$
\left\langle f, 2, a_{P L / O}\right\rangle \subset \pi_{8 j+8+\epsilon}(P L / O)
$$

is defined. As explained in section 2.3 we view the $\alpha$-invariant as a map $\pi_{*}(P L / O) \rightarrow K O_{*}$ by applying the canonical map $p: P L / O \rightarrow G / O$. 
3.1 Theorem. $\alpha\left(\left\langle f, 2, a_{P L / O}\right\rangle\right)=\{1\} \subset K O_{8 j+\epsilon}=\{0,1\}$.

An ingredient in the proof of Theorem 3.1 is the following well-known lemma.

3.2 Lemma. The $\alpha$-invariant $\pi_{8}(G / O) \rightarrow K O_{8}$ is surjective.

Proof. By its geometric definition, the $\alpha$-invariant of $x \in \pi_{8}(G / O)$ in $K O_{8}=\mathbb{Z}$ is calculated as the $\hat{A}$-class of the stable vector bundle over $S^{8}$ classified by $x$. Hence the $\alpha$-invariant only depends on the image of $x$ in $\pi_{8}(B O)$, which is an infinite cyclic group generated say by $t \in \pi_{8}(B O) \cong \mathbb{Z}$. Now the image of $\pi_{8}(G / O)$ in $\pi_{8}(B O)$ is precisely the kernel of the $J$-homomorphism, which is generated by $240 t[21,6.26]$. But the second Pontryagin class of $t$ in $H^{8}\left(S^{8} ; \mathbb{Z}\right)$ is $\pm 6 \cdot\left[S^{8}\right]$ by $\left[16\right.$ where we write $\left[S^{8}\right]$ for a generator of $H^{8}\left(S^{8} ; \mathbb{Z}\right) \cong \mathbb{Z}$. Therefore the $\hat{A}$-class of $t$ computes as

$$
\hat{A}_{2}(t)=\frac{1}{2^{7} \cdot 3^{2} \cdot 5}\left(-4 p_{2}(t)+7 p_{1}(t)^{2}\right)=-\frac{ \pm 4 \cdot 6\left[S^{8}\right]}{2^{7} \cdot 3^{2} \cdot 5}=\mp \frac{1}{240}\left[S^{8}\right],
$$

compare [20, p. 231]. Hence the $\hat{A}$-class of $x=240 t$ is equal to $\mp 1 \cdot\left[S^{8}\right]$, i.e. a generator of $K O_{8} \cong \mathbb{Z}$ is in the image of the $\alpha$-invariant, as we have claimed.

We will also use the following well-known calculations from the surgery exact sequence for homotopy spheres (see e.g. [21, Chapter 6]):

1. $p_{*}: \pi_{7}(P L / O) \rightarrow \pi_{7}(G / O)$ is the zero map.

2. $p_{*}: \pi_{8}(P L / O) \rightarrow \pi_{8}(G / O)$ is isomorphic to the inclusion $\mathbb{Z} / 2 \rightarrow \mathbb{Z} \oplus \mathbb{Z} / 2$.

3. $\pi_{8}(G / P L)=\mathbb{Z}$ and $\pi_{9}(G / P L)=0$.

Proof of Theorem [3.1. Since $a_{P L / O} \in \pi_{7}(P L / O)$ is of order two, it has a lift to some $\bar{a} \in \pi_{7}^{M}(P L / O)$ which we can further map to $p(\bar{a}) \in \pi_{7}^{M}(G / O)$. But $p\left(a_{P L / O}\right)=0 \in \pi_{7}(G / O)$ by calculation 1 so we may choose a lift of $p(a)$ to an element

$$
\delta\left(a_{P L / O}\right) \in \pi_{8}(G / O) \cong \mathbb{Z} \oplus \mathbb{Z} / 2 .
$$

With these definitions, any element in the Toda bracket $\left\langle f, 2, a_{P L / O}\right\rangle$ is of the form $\bar{a} \cdot \overline{S f}$, hence its image in $G / O$ is of the form

$$
p(\bar{a}) \cdot \overline{S f}=\delta\left(a_{P L / O}\right) \cdot S f
$$

for a specific choice of $\delta\left(a_{P L / O}\right)$.

We proceed to calculate the $\alpha$-invariant of $\delta\left(a_{P L / O}\right)$. To do this, we first show that the residue class of $\delta\left(a_{P L / O}\right)$ in

$$
C:=\pi_{8}(G / O) /\left(2, p_{*} \pi_{8}(P L / O)\right)
$$

is non-trivial. Indeed, this residue class is precisely the image of

$$
a_{P L / O} \in \operatorname{ker}\left(p:{ }_{2} \pi_{7}(P L / O) \rightarrow{ }_{2} \pi_{7}(G / O)\right)
$$

under the connecting map for the snake lemma, applied to the diagram

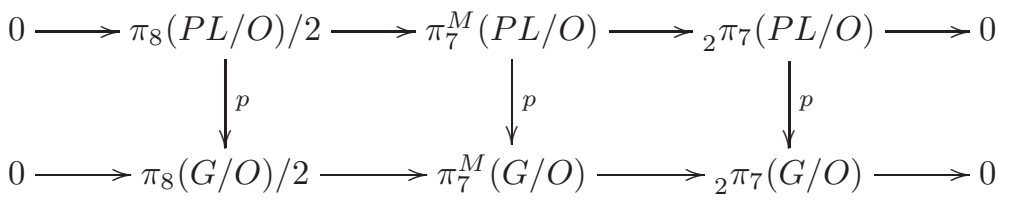


coming from (8).

As a consequence of sequence (8) applied to $G / P L$ together with calculation 3. we have $\pi_{8}^{M}(G / P L)=0$. By the Puppe sequence for the fibration sequence $P L / O \rightarrow G / O \rightarrow G / P L$, the middle vertical map in diagram (10) is therefore injective. The snake lemma implies that the connecting map of the snake lemma is injective, so the residue class of $\delta\left(a_{P L / O}\right)$ in $C$ is non-zero as claimed.

Next we note that the $\alpha$-invariant $\pi_{8}(G / O) \rightarrow K O_{8}=\mathbb{Z}$ becomes zero after restricting to the torsion group $\pi_{8}(P L / O)$. So it induces a well-defined map

$$
C \rightarrow K O_{8} / 2=\mathbb{Z} / 2,
$$

which, in view of Lemma 3.2, is surjective. Indeed it is bijection as Calculation 2 above implies that $C \cong \mathbb{Z} / 2$. We conclude that the $\alpha$-invariant of $\delta\left(a_{P L / O}\right)$ is odd.

Now it follows from Lemma 2.5 that the $\alpha$-invariant of $\delta\left(a_{P L / O}\right) \cdot S f$ in $K O_{8 j+\epsilon}=\mathbb{Z} / 2$ is non-zero, in view of the ring structure of $K O_{*}$ and our assumption that $\alpha(f)=1$. But any element of the Toda bracket was of this form.

Proof of Theorem [1.11. As

$$
S_{*}\left\langle f, 2, a_{P L_{6} / O_{6}}\right\rangle \subset\left\langle f, 2, a_{P L / O}\right\rangle \subset \pi_{8 j+\epsilon}(P L / O),
$$

part (i) follows directly from Theorem 3.1. We proceed to show (ii).

As pointed out in section 2.2. every element $g \in\left\langle f, 2, a_{P L_{6} / O_{6}}\right\rangle$ is realised as a composition

$$
g=\bar{a}_{P L_{6} / O_{6}} \circ \overline{S f}: S^{8 j+\epsilon} \rightarrow M_{7} \rightarrow X,
$$

for specific choices of $\bar{a}_{P L_{6} / O_{6}}$ and $\overline{S f}$, where $\bar{a}_{P L_{6} / O_{6}}$ extends $a_{P L_{6} / O_{6}}$ over $M_{7}$, and $c \circ \overline{S f}=S f$ where $c: M_{7} \rightarrow S^{8}$ is the map collapsing the 7-cell to a point. That is, $g$ is the image of $\bar{a}_{P L_{6} / O_{6}}$ under the map

$$
(\overline{S f})^{*}: \pi_{7}^{M}\left(P L_{6} / O_{6}\right) \rightarrow \pi_{8 j+\epsilon}\left(P L_{6} / O_{6}\right), \quad \bar{b} \mapsto \bar{b} \circ \overline{S f} .
$$

Since $f=S f^{\prime}$ where $2 f^{\prime}=0 \in \pi_{8 j-2+\epsilon}\left(S^{6}\right)$, we can and do choose the map $\overline{S f}$ to be the suspension of a map $S^{8 j-1+\epsilon} \rightarrow M_{6}$. In this case the map $\overline{S f}: S^{8 j+\epsilon} \rightarrow M_{7}$ is a map of co- $H$-spaces and so $(\overline{S f})^{*}$, defined in (12) above, is a group homomorphism.

Now $a_{P L_{6} / O_{6}}$ is divisible by 2 so $\eta \circ a_{P L_{6} / O_{6}}=0$. It follows from Lemma 2.3 that every lift $\bar{a}_{P L_{6} / O_{6}}$ of $a_{P L_{6} / O_{6}}$ has order 2 . It follows that $g$, as the homomorphic image of $\bar{a}_{P L_{6} / O_{6}}$, has order 2 .

In Sections 3.2 and 4 below we will repeat the arguments of Theorem 3.1 and the proof of Theorem 1.11 replacing $P L_{6} / O_{6}$ with other spaces. To avoid repetition we summarise these arguments as follows. Let $h: X \rightarrow P L / O$ be a map. Abusing notation define $\alpha_{X}:=\alpha_{P L / O} \circ h: X \rightarrow K O$.

3.3 Theorem. Suppose that $a \in 2 \pi_{7}(X)$ satisfies $2 a=0$ and $h_{*}(a) \neq 0$. Then $\alpha_{X *}: \pi_{*}(X) \rightarrow K O_{*}$ is split onto in degrees $* \equiv 1,2(\bmod 8)$ and $*>2$. 
Proof. If $X$ is not simply connected we can and do replace $X$ by its universal cover. Since $2 a=0$ we can form the Toda bracket $\langle f, 2, a\rangle \subset \pi_{8 j+\epsilon}(X)$. By naturality of Toda brackets and the $\alpha$ invariant

$$
\alpha(\langle f, 2, a\rangle)=\alpha\left(\left\langle f, a, h_{*}(a)\right)\right\rangle=\{1\},
$$

where the last equality holds by Theorem 3.1. The proof of Theorem 1.11 only used that $a_{P L_{6} / O_{6}} \in 2 \pi_{7}\left(P L_{6} / O_{6}\right)$, so it may be repeated with $a \in 2 \pi_{7}(X)$ to show that $\langle f, 2, a\rangle$ contains an element of order two. This completes the proof.

\subsection{The $\alpha$-invariant on $\pi_{*}\left(P L_{6}\right)$}

In this subsection we prove Theorem 1.14 which states that the $\alpha$-invariant

$$
\alpha: \pi_{8 j+\epsilon}\left(P L_{m}\right) \rightarrow K O_{8 j+\epsilon}
$$

is a split surjection for all $j \geq 1, m \geq 6$ and $\epsilon \in\{1,2\}$. Let $v: P L \rightarrow P L / O$ be the natural map and for $d \in \mathbb{Z}$, let $\rho_{d}: \mathbb{Z} \rightarrow \mathbb{Z} / d$ denote reduction $\bmod d$. The following lemma is well-known.

3.4 Lemma. The homomorphism $v_{*}: \pi_{7}(P L) \rightarrow \pi_{7}(P L / O)$ is isomorphic to the surjection $\rho_{7} \oplus \mathrm{id}: \mathbb{Z} \oplus \mathbb{Z} / 4 \longrightarrow \mathbb{Z} / 7 \oplus \mathbb{Z} / 4$.

Proof. The computation of $\pi_{7}(S P L) \cong \mathbb{Z} \oplus \mathbb{Z} / 4$ is found in [39. p. 29]; see also [7. Remark 4.9]. That $v_{*}: \pi_{*}(P L) \rightarrow \pi_{*}(P L / O)$ is onto follows from [17, Theorem 3.1] and [21, Theorem 6.48].

3.5 Lemma. The stablisation map $S_{*}: \pi_{7}\left(S P L_{6}\right) \rightarrow \pi_{7}(S P L)$ is isomorphic to the inclusion $(\times 4) \oplus \mathrm{id}: \mathbb{Z} \oplus \mathbb{Z} / 4 \longrightarrow \mathbb{Z} \oplus \mathbb{Z} / 4$.

After we use it to prove Theorem 1.14, the proof of Lemma 3.5 will occupy the remainder of the subsection.

Proof of Theorem 1.14. By Lemmas 3.4 and 3.5, the group $2 \pi_{7}\left(S P L_{6}\right)$ has a unique element of order two which maps to $a_{P L_{6} / O_{6}}$ under the composition $P L_{6} \rightarrow P L \rightarrow P L / O$. The theorem now follows from Theorem 3.3 .

For the proof of Lemma 3.5 we require the following two lemmas. They are presumably well-known; we include proofs for completeness.

3.6 Lemma. The homomorphism $\pi_{7}(P L) \rightarrow \pi_{7}(G)$ is isomorphic to the surjection

$$
\rho_{240}+(\times 60): \mathbb{Z} \oplus \mathbb{Z} / 4 \longrightarrow \mathbb{Z} / 240 .
$$

Proof. We consider the fibration $P L \rightarrow G \rightarrow G / P L$ and the following part of its homotopy long exact sequence

$$
\pi_{8}(G / P L) \rightarrow \pi_{7}(P L) \rightarrow \pi_{7}(G) \rightarrow \pi_{7}(G / P L) .
$$

Since $\pi_{7}(G / P L)=0=\pi_{9}(G / P L)$ and $\pi_{8}(G / P L) \cong \mathbb{Z}$ by surgery theory (see e.g. [21, 6.48]), this sequence must be isomorphic to

$$
0 \rightarrow \mathbb{Z} \rightarrow \mathbb{Z} \oplus \mathbb{Z} / 4 \stackrel{y+z}{\longrightarrow} \mathbb{Z} / 240 \rightarrow 0 .
$$


Since $y+z$ is surjective, $y$ is isomorphic to $\rho_{240}$ via an automorphism of $\mathbb{Z} / 240$. Since $y+z$ has a free kernel, $z$ must be injective and is thus isomorphic, via an automorphism of $\{0\} \oplus \mathbb{Z} / 4$, to multiplication by 60 on residue classes. The lemma now follows.

3.7 Lemma. For $k \leq 2 n-5$ the homomorphism $I: \pi_{k}\left(G_{n}\right) \rightarrow \pi_{k+n}\left(S^{n}\right)$ is an isomorphism, for $k=2 n-4$ it is surjective.

Proof. Let $F_{n-1} \subset G_{n}$ be the submonoid of base-point preserving maps $S^{n-1} \rightarrow$ $S^{n-1}$. There is a fibration sequence $F_{n-1} \rightarrow S G_{n} \stackrel{\text { ev }}{\longrightarrow} S^{n-1}$, where ev if given by evaluation at the base-point [22, Lemma 3.1]. It is well-known that the homotopy long exact sequences of these fibrations fit into the following commutative diagram:

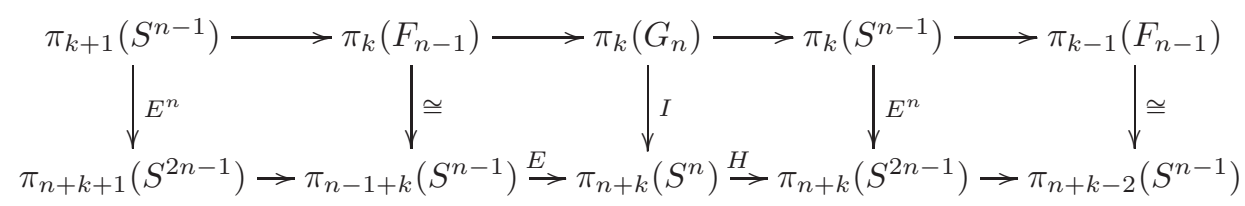

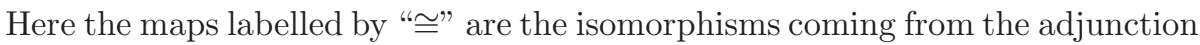
between based suspension and based loop space and the bottom row is part of the EHP sequence [38, p. 548]. The vertical suspension maps $E^{n}$ are isomorphisms if $k \leq 2 n-5$ and so $I: \pi_{k}\left(G_{n}\right) \rightarrow \pi_{k+n}\left(S^{n}\right)$ is an isomorphism by the 5-Lemma. If $k=2 n-4$ only the right-most map $E^{n}$ is an isomorphism, and the 5-Lemma (rather the 4-Lemma) implies that $I$ is surjective.

Proof of Lemma 3.5. We compare $\pi_{*}\left(P L_{6}\right)$ with the homotopy groups of $\widetilde{P L_{6}}$, the semi-simplicial group of block automorphisms of $\mathbb{R}^{6}$. By [9, Proposition 5.6], the map $P L_{6} \rightarrow \widetilde{P L}_{6}$ induces an isomorphism $\pi_{7}\left(P L_{6}\right) \rightarrow \pi_{7}\left(\widetilde{P L}_{6}\right)$. Hence we consider the group $\pi_{7}\left(\widetilde{P L}_{6}\right)$ which lies in the following commutative diagram of exact sequences:

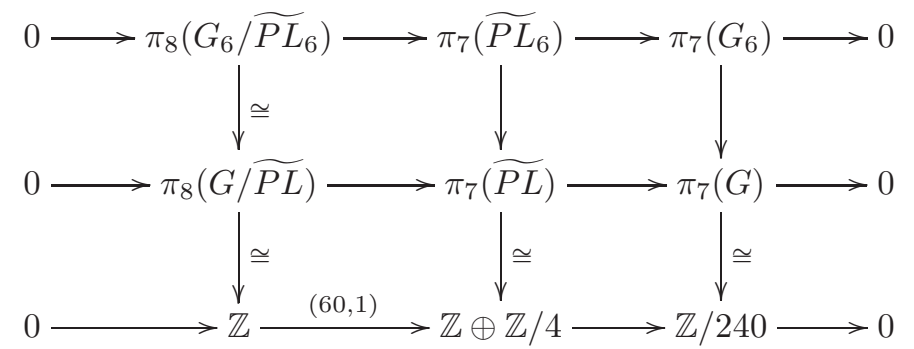

The isomorphism between the bottom two sequences follows since in the limit $P L \rightarrow \widehat{P L}$ is an equivalence 9] and the isomorphisms for $P L$ appeared in the proof of Lemma [3.6. Now the natural map $\pi_{8}\left(G_{6} / \widetilde{P L_{6}}\right) \rightarrow \pi_{8}(G / \widetilde{P L})$ is an isomorphism (see e.g. [29, Theorem 1.10]). Hence it suffices to prove that $i_{*}: \pi_{7}\left(G_{6}\right) \rightarrow \pi_{7}(G)$ is isomorphic to the inclusion $\mathbb{Z} / 60 \rightarrow \mathbb{Z} / 240$. By Lemma 3.7. the map $I: \pi_{7}\left(G_{6}\right) \rightarrow \pi_{13}\left(S^{6}\right)$ is an isomorphism. It follows that the map $i_{*}: \pi_{7}\left(G_{6}\right) \rightarrow \pi_{7}(G)$ is isomorphic to the stabilisation homomorphism $\pi_{13}\left(S^{6}\right) \rightarrow \pi_{7}^{S}$, which by [33, Propositions 5.15 and 13.6] is isomorphic to the inclusion $\mathbb{Z} / 60 \rightarrow \mathbb{Z} / 240$, as required. 


\section{$4 \operatorname{Im}(J)$-homotopy spheres}

In this section we prove Theorems 1.2 and 1.13 both of which concern $\operatorname{Im}(J)$ homotopy spheres. The definition of $\operatorname{Im}(J)$-homotopy spheres is based on foundational facts about the space $S G$ which we now recall. For a prime $p$ and an $H$-space $X$, recall that $X_{(p)}$ denotes the $p$-localisation of $X$. The map $\phi: S G_{(p)} \times S G_{(p)} \rightarrow S G_{(p)}$ is the $p$-localisation of the multiplication map on $S G$.

4.1 Theorem ([23, V Theorems 4.7 and 4.8]). For each prime $p$ there are spaces $\mathcal{J}_{p}$ and $C_{p}$ and maps $i_{\mathcal{J}_{p}}: \mathcal{J}_{p} \rightarrow S G_{(p)}$ and $i_{C_{p}}: C_{p} \rightarrow S G_{(p)}$ such that the composition

$$
\mathcal{J}_{p} \times C_{p} \stackrel{i_{\mathcal{J}_{p}} \times i_{C_{p}}}{\longrightarrow} S G_{(p)} \times S G_{(p)} \stackrel{\phi}{\longrightarrow} S G_{(p)}
$$

is a weak homotopy equivalence.

The homotopy groups of the spaces $\mathcal{J}_{p}$ are closely related to the image of the $J$-homomorphism $I^{-1} \circ J_{*}: \pi_{i}(S O) \rightarrow \pi_{*}(S G)$ as we now recall. Let $\alpha_{J, p}: \pi_{*}\left(\mathcal{J}_{p}\right) \rightarrow \operatorname{Tors}\left(K O_{*}\right)$ be the restriction of the $\alpha$-invariant on $\pi_{*}(S G)$ to $\pi_{*}\left(\mathcal{J}_{p}\right) \subset \pi_{*}(S G)_{(p)}$. The next lemma follows immediately from 23, Remark 5.6].

4.2 Lemma. The groups $\operatorname{Im}\left(J_{*}\right)_{(p)} \subset \pi_{*}(S G)_{(p)}$ are summands of the groups $\pi_{*}\left(\mathcal{J}_{p}\right)$ and there is a split short exact sequence

$$
0 \rightarrow \operatorname{Im}\left(J_{*}\right)_{(p)} \rightarrow \pi_{*}\left(\mathcal{J}_{p}\right) \stackrel{\alpha_{J, p}}{\longrightarrow} \operatorname{Tors}\left(K O_{*}\right)_{(p)} \rightarrow 0 .
$$

Following [23, $\mathrm{V} \S 4$ ], we define $\mathcal{J}_{\infty}:=\prod_{p} \mathcal{J}_{p}$ and $C_{\infty}:=\prod_{p} C_{p}$ and let

$$
\psi: S G \stackrel{\sim}{\rightarrow} \mathcal{J}_{\infty} \times C_{\infty}
$$

be the weak equivalence stemming from Theorem 4.1 We identify $\pi_{*}(S G)=$ $\pi_{*}\left(\mathcal{J}_{\infty}\right) \times \pi_{*}\left(C_{\infty}\right)$ using the map $\psi$ and then define $\alpha_{J}: \pi_{*}\left(\mathcal{J}_{\infty}\right) \rightarrow \operatorname{Tors}\left(K O_{*}\right)$ to be the restriction of the $\alpha$-invariant on $\pi_{*}(S G)$ to $\pi_{*}\left(\mathcal{J}_{\infty}\right)$.

Let $q: S G \rightarrow G / O$ be the natural map and observe that the isomorphism $I: \pi_{*}(S G) \stackrel{\cong}{\rightarrow} \pi_{*}^{s}$ induces an isomorphism $\bar{I}: \operatorname{Tors}\left(\pi_{*}(G / O)\right) \rightarrow \operatorname{coker}\left(J_{*}\right)$. The splitting $\pi_{*}(S G)=\pi_{*}\left(\mathcal{J}_{\infty}\right) \times \pi_{*}\left(C_{\infty}\right)$ then induces a splitting of $q_{*}$ and of its image as

$$
q_{*}=q_{*}^{J} \times q_{*}^{C}: \pi_{*}\left(\mathcal{J}_{\infty}\right) \times \pi_{*}\left(C_{\infty}\right) \rightarrow q_{*}\left(\pi_{*}\left(\mathcal{J}_{\infty}\right)\right) \times q_{*}\left(\pi_{*}\left(C_{\infty}\right)\right)=\operatorname{coker}\left(J_{*}\right) .
$$

Because $\operatorname{Im}\left(J_{*}\right)$ is contained in $\pi_{*}\left(\mathcal{J}_{\infty}\right)$ it follows that we have an isomorphism $q_{*}^{C}: \pi_{*}\left(C_{\infty}\right) \rightarrow q_{*}\left(\pi_{*}\left(C_{\infty}\right)\right)$, whereas $\alpha_{J}: \pi_{*}\left(\mathcal{J}_{\infty}\right) \rightarrow$ Tors $\left(\mathrm{KO}_{*}\right)$ descends by (13) to an isomorphism $\overline{\alpha_{J}}: q_{*}\left(\pi_{*}\left(\mathcal{J}_{\infty}\right)\right) \rightarrow \operatorname{Tors}\left(K O_{*}\right)$, as $\operatorname{ker}\left(\alpha_{J}\right) \subset \operatorname{ker}\left(q_{*}\right)$. We use the splitting (15) of coker $\left(J_{*}\right)$, induced from the splitting (14) of $S G$ to define a splitting of the $\alpha$-invariant on $\operatorname{coker}\left(J_{*}\right)$ :

$$
s_{*}:=\operatorname{incl} \circ{\overline{\alpha_{J}}}^{-1}: \operatorname{Tors}\left(K O_{*}\right) \rightarrow q_{*}\left(\pi_{*}\left(\mathcal{J}_{\infty}\right)\right) \hookrightarrow \operatorname{coker}\left(J_{*}\right) .
$$

Recalling the Kervaire-Milnor homomorphism $\Phi: \Theta_{n+1} \rightarrow \operatorname{coker}\left(J_{n+1}\right)$ we make the following definition. 
4.3 Definition $\left(\operatorname{Im}(J)\right.$-homotopy spheres). A homotopy sphere $\Sigma \in \Theta_{n+1}$ is an $\operatorname{Im}(J)$-homotopy sphere if

$$
\Phi(\Sigma) \in s_{*}\left(\operatorname{Tors}\left(K O_{n+1}\right)\right) \subset \operatorname{coker}\left(J_{n+1}\right)
$$

and $\Theta_{n+1}^{J} \subset \Theta_{n+1}$ is the subgroup of $\operatorname{Im}(J)$-homotopy spheres.

Since the Kervaire-Milnor sequence $b P_{8 k+2} \rightarrow \Theta_{8 k+1} \rightarrow \operatorname{coker}\left(J_{8 k+1}\right)$ splits by [8. Theorem 1.2], $b P_{8 k+3}=0$ and $\operatorname{Tors}\left(K O_{*}\right)=0$ unless $* \equiv 1,2 \bmod 8$, we have

4.4 Lemma. There is an isomorphism

$$
\Theta_{n+1}^{J} \cong b P_{n+2} \oplus \operatorname{Tors}\left(K O_{n+1}\right)
$$

with $\alpha\left(\Theta_{n+1}^{J}\right)=\operatorname{Tors}\left(K O_{n+1}\right)$ and $\alpha\left(b P_{n+2}\right)=0$.

Let $u: S P L \rightarrow S G$ be the natural map and let $\operatorname{pr}_{C_{\infty}}: S G \rightarrow C_{\infty}$ be the composition of the map $\psi$ of (14) and projection to the second factor.

4.5 Definition. We define $i^{J}: S P L^{J} \subset S P L$ to be the inclusion of the homotopy fiber of the composition

$$
S P L \stackrel{u}{\longrightarrow} S G \stackrel{\mathrm{pr}_{C_{\infty}}}{\longrightarrow} C_{\infty} .
$$

Similarly we define $i_{6}^{J}: S P L_{6}^{J} \subset S P L_{6}$ to be the inclusion of the homotopy fiber of the composition

$$
S P L_{6} \stackrel{S}{\longrightarrow} S P L \stackrel{u}{\longrightarrow} S G \stackrel{\mathrm{pr}_{\infty}}{\longrightarrow} C_{\infty} .
$$

Let $v^{+}: S P L \rightarrow P L / O$ be the restriction of $v: P L \rightarrow P L / O$ to $S P L$.

4.6 Lemma. The image under the canonical maps of $\pi_{*}\left(S P L^{J}\right)$ consists precisely of the $\operatorname{Im}(J)$-homotopy spheres, i.e. we have

$$
\left(\Psi \circ v_{*}^{+} \circ i_{*}^{J}\right)\left(\pi_{*}\left(S P L^{J}\right)\right)=\Theta_{*}^{J} .
$$

Proof. We have that $\Phi \circ \Psi \circ v_{*}^{+}\left(\operatorname{Im}\left(i_{*}^{J}\right)\right) \subset q_{*}\left(\pi_{*}\left(\mathcal{J}_{\infty}\right)\right)$ by naturality and because $u_{*}\left(\operatorname{Im}\left(i_{*}^{J}\right)\right) \subset \pi_{*}\left(\mathcal{J}_{\infty}\right)$ by the splitting of $S G$. By definition of $\operatorname{Im}(J)$-spheres therefore the left hand side is contained in the set of $\operatorname{Im}(J)$-spheres.

It remains to show that every $\operatorname{Im}(J)$-sphere is contained in the left-hand side. First, we look at the summand $b P_{*+1} \subset \Theta_{*}^{J}$. Recall that the natural map $\pi_{*}(S P L) \rightarrow \pi_{*}(P L / O)$ is onto, corresponding to the fact that the stable tangent bundle of every homotopy sphere is trivial (see e. g. 21, Theorem 6.45]). Every $b P$-sphere is mapped by $\Phi$ to 0 in $\operatorname{coker}\left(J_{*}\right)$, therefore, using the splitting (15) of $q_{*}$ and naturality, every lift of it to $\pi_{*}(S P L)$ is mapped to $\pi_{*}\left(\mathcal{J}_{\infty}\right)$ under $u_{*}$ and consequently lies in the image of $\pi_{*}\left(S P L^{J}\right)$.

Because of Lemma 4.4. it remains to find one sphere with $\alpha$-invariant 1 for each relevant dimension $8 k+1$ and $8 k+2$ in the left hand side. We have to show that the restriction of the alpha-invariant to $u_{*}^{-1}\left(\pi_{*}\left(\mathcal{J}_{\infty}\right)\right)$ surjects onto Tors $\left(\mathrm{KO}_{*}\right)$. Note, however, that the cokernel of $\pi_{*}(S P L) \rightarrow \pi_{*}(S G)$ is the kernel of $\pi_{*}(S G) \rightarrow \pi_{*}(G / P L)$. Via the Kervaire-Milnor braid (see e.g. 21, Theorem 6.48]) the latter map can be identified with the Kervaire invariant which is known to be zero except for some dimensions $*=8 k+6$ (compare [21, Corollary 6.43]). But these dimensions are not relevant for us as in those dimensions Tors $\left(K O_{*}\right)=0$. Therefore $u_{*}$ is surjective in the relevant dimensions, and because $\alpha\left(\pi_{*}\left(\mathcal{J}_{\infty}\right)\right)=\operatorname{Tors}\left(\mathrm{KO}_{*}\right)$ we are done. 
We require the following lemmas to prove Theorems 1.13 and 1.2 . We defer their proofs to the end of the section.

4.7 Lemma. The map $i_{6 *}^{J}: \pi_{7}\left(S P L_{6}^{J}\right) \rightarrow \pi_{7}\left(S P L_{6}\right)$ is an isomorphism.

4.8 Lemma (C.f. [1, Theorem 12.18]). Let $g \in \pi_{8 j+1}\left(\mathcal{J}_{2}\right)$ have $\alpha(g)=1$. Then

$$
\left\langle\eta_{8 j+1}, 2_{8 j+1}, g\right\rangle \subset\{2,6\} \subset \pi_{8 j+3}\left(\mathcal{J}_{2}\right) \cong \mathbb{Z} / 8 .
$$

Proof of Theorem 1.13. From Lemmas 3.4, 3.5 and 4.7 we deduce that there is an element $a_{S P L_{6}^{J}} \in 2 \pi_{7}\left(S P L_{6}^{J}\right)$ which maps to $a_{P L_{6} / O_{6}} \in \pi_{7}\left(P L_{6} / O_{6}\right)$ under the map induced by the composition $S P L_{6}^{J} \rightarrow S P L_{6} \rightarrow P L_{6} / O_{6}$. The theorem follows from Theorem 3.3 .

Proof of Theorem 1.2. The proof of Theorem 1.13, shows that there is an element $g_{S P L_{6}^{J}}$ of order two in the Toda bracket $\left\langle f, 2, a_{S P L_{6}^{J}}\right\rangle \subset \pi_{8 j+1}\left(S P L_{6}^{J}\right)$, so that $\alpha\left(g_{S P L_{6}^{J}}\right)=1$. Choose an element

$$
e \in\left\langle\eta_{8 j+1}, 2_{8 j+1}, g_{S P L_{6}^{J}}\right\rangle \subset \pi_{8 j+3}\left(S P L_{6}^{J}\right)
$$

and consider the following diagram:

$$
\begin{aligned}
& \pi_{8 j+3}\left(S P L_{6}^{J}\right) \stackrel{i_{6 *}^{J}}{\longrightarrow} \pi_{8 j+3}\left(S P L_{6}\right) \stackrel{\pi_{S P L+3}}{\longrightarrow} \pi_{8 j+3}(S P L) \stackrel{\widehat{q}_{S P L *}}{\longrightarrow} \pi_{8 j+3}\left(J_{2} \times C_{2}\right) \\
& \underset{\pi_{8 j+3}\left(P L_{6} / O_{6}\right) \stackrel{S_{P L / O *}}{\longrightarrow} \pi_{8 j+3}(P L / O)}{\downarrow}
\end{aligned}
$$

By Lemma 4.8

$$
\widehat{q}_{S P L *} \circ S_{S P L *} \circ i_{*}(e) \in\{2,6\} \oplus\{0\} \subset \pi_{8 j+3}\left(J_{2} \times C_{2}\right)
$$

and by a theorem of Brumfiel [7. Theorem 1.4]

$$
\pi_{8 j+3}(S P L) \cong \mathbb{Z} \oplus \mathbb{Z} / 8 \oplus \operatorname{coker}\left(J_{8 j+3}\right)
$$

Since $e$ is 2-primary torsion, it follows that

$$
S_{*}(e) \in\{0\} \oplus\{2,6\} \oplus\{0\} .
$$

As $\pi_{8 j+3}(S O) \cong \mathbb{Z}$ by Bott periodicity, and $\pi_{8 j+3}(P L / O) \cong \Theta_{8 j+3}$ is finite by [17, the torsion of $\pi_{8 j+3}(S P L)$ injects into $\pi_{8 j+3}(P L / O)$. Since the $\mathbb{Z} / 8$-summand maps trivially to $\operatorname{coker}\left(J_{8 j+3}\right)$, e must map into the subgroup $\Psi\left(b P_{8 j+4}\right) \subset \pi_{8 j+3}(P L / O)$, and hence to a generator of $\Psi\left({ }_{4} b P_{8 j+3}\right)$. The commutativity of diagram (16) above shows that $e \in \operatorname{Im}\left(S_{P L_{6} / O_{6} *}\right)$, which proves the theorem.

Proof of Lemma 4.7. To see that $i_{6 *}^{J}: \pi_{7}\left(S P L_{6}^{J}\right) \rightarrow \pi_{7}\left(S P L_{6}\right)$ is an isomorphism, we recall that by the definition of $S P L_{6}^{J}$ we have a commutative diagram 
of fibrations

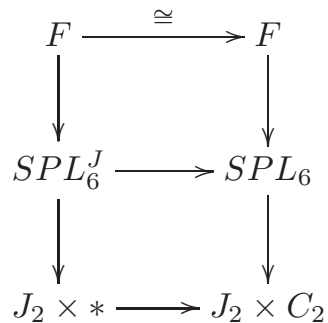

where $F$ is the homotopy fiber of the map $S P L_{6} \rightarrow S P L \rightarrow \mathcal{J}_{2} \times C_{2}$. Now $\pi_{7}^{s}=\operatorname{Im}\left(J_{7}\right)$ and so $\pi_{7}\left(C_{2}\right)=0$, and so diagram chasing in the ladder made by the homotopy long exact sequences of the above fibrations gives the result, provided that we can prove that the map $\pi_{8}\left(S P L_{6}\right) \rightarrow \pi_{8}\left(\mathcal{J}_{2} \times C_{2}\right)$ is onto, and we do this now. We have $\pi_{8}\left(\mathcal{J}_{2} \times C_{2}\right) \cong \pi_{8}^{s} \cong(\mathbb{Z} / 2)^{2}$ and by 33. Theorem 7.1] the stablisation homomorphism $\pi_{14}\left(S^{6}\right) \rightarrow \pi_{8}^{s}$ is onto. By Lemma 3.7 $J: \pi_{8}\left(G_{6}\right) \rightarrow \pi_{14}\left(S^{6}\right)$ is onto and by [9, Proposition 5.6] the map $\pi_{8}\left(\widetilde{P L}_{6}\right) \rightarrow$ $\pi_{8}\left(P L_{6}\right)$ is onto. Hence it is enough to show that $\pi_{8}\left(\widetilde{P L_{6}}\right) \rightarrow \pi_{8}\left(G_{6}\right)$ is onto. But this follows from the exact sequence

$$
\cdots \rightarrow \pi_{8}\left(\widetilde{P L}_{6}\right) \rightarrow \pi_{8}\left(G_{6}\right) \rightarrow \pi_{8}\left(G_{6} / \widetilde{P L}_{6}\right) \stackrel{\partial}{\longrightarrow} \pi_{7}\left(\widetilde{P L}_{6}\right) \rightarrow \ldots
$$

since the boundary map $\partial: \pi_{8}(G / \widetilde{P L}) \rightarrow \pi_{7}(\widetilde{P L}) \cong \pi_{7}(P L)$ is injective and $\pi_{8}\left(G_{6} / \widetilde{P L_{6}}\right) \cong \pi_{8}(G / \widetilde{P L})$ (we saw both assertions in the proof of Lemma 3.5).

Proof of Lemma 4.8. In [1, Proposition 12.18] Adams proves that the $e$-invariant of the Toda bracket $\left\langle\eta, 2, \mu_{8 j+1}\right\rangle$ is the set $\left\{\frac{1}{4}, \frac{-1}{4}\right\} \in \mathbb{Q} / \mathbb{Z}$. By [23, Remark 5.6] the $e$-invariant gives a split surjection from $\left(\pi_{*}^{s}\right)_{(2)}$ onto $\pi_{*}\left(J_{2}\right)$, proving the lemma.

\section{A The Gromoll filtration: table of values}

We think that our results about the Gromoll filtration and the existence of elements rather deep down with non-trivial $\alpha$-invariant are interesting in their own right. In this appendix we place them in context by assembling some results from the literature about the Gromoll filtration. This is an update of the corresponding table in [10, Appendix A]. Recall $\Gamma_{b P}^{4 i-1}=\Sigma^{-1}\left(b P_{4 i}\right) \subseteq \Gamma^{4 i-1}$, let $f_{M} \in \Gamma_{b P}^{4 i-1}$ be the generator corresponding to the Milnor sphere and define the group $\Gamma_{(k) b P}^{4 i-1}:=\Gamma_{(k)}^{4 i-1} \cap \Gamma_{b P}^{4 i-1}$. In the following table, the new results of the current article are printed red. 


\begin{tabular}{|c|c|}
\hline$\Gamma_{(5)}^{7} \cong \mathbb{Z} / 28$ & $\begin{array}{l}\Gamma_{(5)}^{7} \neq \Gamma_{(4)}^{7} \supset 0=\Gamma_{(3)}^{7} \text {. The inequality for } \Gamma_{(4)}^{7} \neq \\
\Gamma_{(5)}^{7} \text { is due to Weiss [36] who proved that } \Gamma_{(4)}^{7} \text { has } \\
\text { at most } 14 \text { elements. }\end{array}$ \\
\hline$\Gamma_{(6)}^{8} \cong \mathbb{Z} / 2$ & nothing known \\
\hline$\Gamma_{(7)}^{9} \cong(\mathbb{Z} / 2)^{3}$ & $\Gamma_{(6)}^{9} \supset \mathbb{Z} / 2, \alpha\left(\Gamma_{(6)}^{9}\right)=\mathbb{Z} / 2$ by Theorem 1.1 \\
\hline$\Gamma_{(8)}^{10} \cong \mathbb{Z} / 6$ & $\Gamma_{(6)}^{10} \supset \mathbb{Z} / 2, \alpha\left(\Gamma_{(6)}^{10}\right)=\mathbb{Z} / 2$ by Theorem 1.1 \\
\hline$\Gamma_{(9)}^{11} \cong \mathbb{Z} / 992$ & $\Gamma_{(8)}^{11} \subset \mathbb{Z} / 496$ by [35], $\Gamma_{(6)}^{11} \supset \mathbb{Z} / 4$ by Theorem $[1.2$ \\
\hline \multicolumn{2}{|l|}{$\Gamma_{(10)}^{12}=0$} \\
\hline$\Gamma_{(11)}^{13} \cong \mathbb{Z} / 3$ & $\Gamma_{(11)}^{13}=\Gamma_{(10)}^{13}=\Gamma_{(9)}^{13}$ by $[4]$ \\
\hline$\Gamma_{(12)}^{14} \cong \mathbb{Z} / 2$ & nothing known \\
\hline$\Gamma_{(13)}^{15} \cong \mathbb{Z} / 2 \oplus \mathbb{Z} / 8128$ & $\Gamma_{(12)}^{15} \cong \mathbb{Z} / 2 \oplus \mathbb{Z} / 4064$ by [4, 35] \\
\hline$\Gamma_{(14)}^{16} \cong \mathbb{Z} / 2$ & nothing known, conjecturally $\Gamma_{(13)}^{16}=0$ \\
\hline$\Gamma_{(15)}^{17} \cong(\mathbb{Z} / 2)^{2}$ & $\Gamma_{(6)}^{17} \supset \mathbb{Z} / 2, \alpha\left(\Gamma_{(6)}^{17}\right)=\mathbb{Z} / 2$ by Theorem 1.1 \\
\hline$\Gamma_{(16)}^{18} \cong \mathbb{Z} / 8 \oplus \mathbb{Z} / 2$ & $\begin{array}{l}\text { By Theorem } 1.1, \alpha\left(\Gamma_{(6)}^{18}\right)=\mathbb{Z} / 2 \text {. Because } \mathbb{Z} / 8= \\
\operatorname{ker}(\alpha), \Gamma_{(6)}^{18} \supset\{0\} \oplus \mathbb{Z} / 2 .\end{array}$ \\
\hline$\Gamma^{8 j+1}, j \geq 1$ & $\Gamma_{(6)}^{8 j+1} \supset \mathbb{Z} / 2, \alpha\left(\Gamma_{(6)}^{8 j+1}\right)=\mathbb{Z} / 2$ by Theorem 1.1 \\
\hline$\Gamma^{8 j+2}, j \geq 1$ & $\Gamma_{(6)}^{8 J+2} \supset \mathbb{Z} / 2, \alpha\left(\Gamma_{(6)}^{8 j+2}\right)=\mathbb{Z} / 2$ by Theorem 1.1 \\
\hline$\Gamma_{b P}^{8 j+3}, j \geq 1$ & $\Gamma_{(6) b P}^{8 j+3} \supset \mathbb{Z} / 4$ by Theorem 1.2 \\
\hline$\Gamma_{b P}^{4 i-1}, i \geq 4$ & $\Gamma_{(2 i+1) b P}^{4 i-1} \neq 0$ by [4, Theorem 1.1$]$ \\
\hline$\Gamma_{b P}^{4 i-1}, i \geq 2$ & $f_{M} \notin \Gamma_{(4 i-4) b P}^{4 i-1}$ by [35, 2nd Corollary, p. 888] \\
\hline
\end{tabular}

\section{References}

[1] J. F. Adams, On the groups $J(X)$. IV, Topology 5 (1966), 21-71. MR0198470 10 1 1.3 3.14 .8 4

[2] Bernd Ammann, Mattias Dahl, and Emmanuel Humbert, Surgery and harmonic spinors, Adv. Math. 220 (2009), no. 2, 523-539, DOI 10.1016/j.aim.2008.09.013. MR2466425 11.7

[3] _ Harmonic spinors and local deformations of the metric, Math. Res. Lett. 18 (2011), no. 5, 927-936, DOI 10.4310/MRL.2011.v18.n5.a10. MR2875865 个

[4] P. Antonelli, D. Burghelea, and P. J. Kahn, Gromoll groups, Diff $S^{n}$ and bilinear constructions of exotic spheres, Bull. Amer. Math. Soc. 76 (1970), 772-777. MR0283809 11 A

[5] Christian Bär, Metrics with harmonic spinors, Geom. Funct. Anal. 6 (1996), no. 6, 899 942, DOI 10.1007/BF02246994. MR1421872 1.1

[6] Boris Botvinnik, Johannes Ebert, and Oscar Randal-Williams, Infinite loop spaces and positive scalar curvature, Invent. Math. (2017), 1-87. 11.2

[7] G. Brumfiel, On the homotopy groups of BPL and PL/O, Ann. of Math. (2) 88 (1968), 291-311. MR0234458 1.4 3.24

[8] , On the homotopy groups of BPL and PL/O. II, Topology 8 (1969), 305-311. MR0248830 喵

[9] Dan Burghelea and Richard Lashof, The homotopy type of the space of diffeomorphisms. I, II, Trans. Amer. Math. Soc. 196 (1974), 1-36; ibid. 196 (1974), 37-50. MR0356103 回 $1 . 3 \longdiv { 3 . 2 4 }$

[10] Diarmuid Crowley and Thomas Schick, The Gromoll filtration, KO-characteristic classes and metrics of positive scalar curvature, Geom. Topol. 17 (2013), no. 3, 1773-1789, DOI 10.2140/gt.2013.17.1773. MR3073935 101.3 1.3 2.1] 3.1]

[11] Johannes Ebert, The two definitions of the index difference, Trans. Americ. Math. Soc. 369 (2017), no. 10, 7469-7507. 11.1 
[12] Søren Galatius and Oscar Randal-Williams, Stable moduli spaces of high-dimensional manifolds, Acta Math. 212 (2014), no. 2, 257-377, DOI 10.1007/s11511-014-0112-7. MR3207759 1.5

[13] Detlef Gromoll, Differenzierbare Strukturen und Metriken positiver Krümmung auf Sphären, Math. Ann. 164 (1966), 353-371 (German). MR0196754 11

[14] Bernhard Hanke, Thomas Schick, and Wolfgang Steimle, The space of metrics of positive scalar curvature, Publ. Math. Inst. Hautes Études Sci. 120 (2014), 335-367, DOI 10.1007/s10240-014-0062-9. MR3270591 1.2

[15] Nigel Hitchin, Harmonic spinors, Advances in Math. 14 (1974), 1-55. MR0358873 11.1 1.11 .10

[16] Michel A. Kervaire, A note on obstructions and characteristic classes, Amer. J. Math. 81 (1959), 773-784. MR0107863 3.1

[17] Michel A. Kervaire and John W. Milnor, Groups of homotopy spheres. I, Ann. of Math. (2) 77 (1963), 504-537. MR0148075 11.3 1.4 3.2 4

[18] Robion C. Kirby and Laurence C. Siebenmann, Foundational essays on topological manifolds, smoothings, and triangulations, Princeton University Press, Princeton, N.J.; University of Tokyo Press, Tokyo, 1977. With notes by John Milnor and Michael Atiyah; Annals of Mathematics Studies, No. 88. MR0645390 12

[19] Timothy Lance, Differentiable structures on manifolds, Surveys on surgery theory, Vol. 1, Ann. of Math. Stud., vol. 145, Princeton Univ. Press, Princeton, NJ, 2000, pp. 73-104. MR1747531 1.3

[20] H. Blaine Lawson Jr. and Marie-Louise Michelsohn, Spin geometry, Princeton Mathematical Series, vol. 38, Princeton University Press, Princeton, NJ, 1989. MR1031992 3.1

[21] Wolfgang Lück, A basic introduction to surgery theory, Topology of high-dimensional manifolds, No. 1, 2 (Trieste, 2001), ICTP Lect. Notes, vol. 9, Abdus Salam Int. Cent. Theoret. Phys., Trieste, 2002, pp. 1-224. MR1937016 3.1 3.2 3.2 4

[22] Ib Madsen and R. James Milgram, The classifying spaces for surgery and cobordism of manifolds, Annals of Mathematics Studies, vol. 92, Princeton University Press, Princeton, N.J.; University of Tokyo Press, Tokyo, 1979. MR548575 13.2

[23] J. Peter May, $E_{\infty}$ ring spaces and $E_{\infty}$ ring spectra, Lecture Notes in Mathematics, Vol. 577, Springer-Verlag, Berlin-New York, 1977. With contributions by Frank Quinn, Nigel Ray, and Jørgen Tornehave. MR0494077 1.4 1.4 4.1 4, 4 4

[24] J. P. May, The spectra associated to $\mathcal{I}$-monoids, Math. Proc. Cambridge Philos. Soc. 84 (1978), no. 2, 313-322. MR0488033 1.4

[25] John W. Milnor, Remarks concerning spin manifolds, Differential and Combinatorial Topology (A Symposium in Honor of Marston Morse), Princeton Univ. Press, Princeton, N.J., 1965, pp. 55-62. MR0180978 11

[26] Claude Morlet, Isotopie et pseudo-isotopie, C. R. Acad. Sci. Paris Sér. A-B 266 (1968), A559-A560 (French). MR0236935 11.3

[27] Joseph A. Neisendorfer, Homotopy groups with coefficients, J. Fixed Point Theory Appl. 8 (2010), no. 2, 247-338, DOI 10.1007/s11784-010-0020-1. MR2739026 2.2 2.2 2.2

[28] Jonathan Rosenberg, $C^{*}$-algebras, positive scalar curvature, and the Novikov conjecture, Inst. Hautes Études Sci. Publ. Math. 58 (1983), 197-212 (1984). MR720934 (85g:58083) 1.2

[29] C. P. Rourke and B. J. Sanderson, Block bundles. III. Homotopy theory, Ann. of Math. (2) 87 (1968), 431-483. MR0232404 13.2

[30] Thomas Schick, A counterexample to the (unstable) Gromov-Lawson-Rosenberg conjecture, Topology 37 (1998), no. 6, 1165-1168, DOI 10.1016/S0040-9383(97)00082-7. MR1632971 (99j:53049) 11.2

[31] The topology of scalar curvature, Proceedings of the International Congress of Mathematicians Seoul 2014, VOLUME II, 2014, pp. 1285-1308. arXiv:1405.4220.

[32] Stephan Stolz, Simply connected manifolds of positive scalar curvature, Ann. of Math. (2) 136 (1992), no. 3, 511-540, DOI 10.2307/2946598. MR1189863 (93i:57033) 
[33] Hirosi Toda, Composition methods in homotopy groups of spheres, Annals of Mathematics Studies, No. 49, Princeton University Press, Princeton, N.J., 1962. MR0143217 13.2 4

[34] Nils Waterstraat, A remark on the space of metrics having nontrivial harmonic spinors, J. Fixed Point Theory Appl. 13 (2013), no. 1, 143-149, DOI 10.1007/s11784-013-0096-5. MR3071945 1.1

[35] Michael Weiss, Sphères exotiques et l'espace de Whitehead, C. R. Acad. Sci. Paris Sér. I Math. 303 (1986), no. 17, 885-888 (French, with English summary). MR870913 (87m:57038) $1 \mathrm{~A}$

[36] , Pinching and concordance theory, J. Differential Geom. 38 (1993), no. 2, 387416. MR1237489 (95a:53057) A

[37] _ Dalian notes on rational Pontrjagin classes, 2016. arXiv: http://arxiv.org/pdf/1507.00153v3.pdf. 11.5

[38] George W. Whitehead, Elements of homotopy theory, Graduate Texts in Mathematics, vol. 61, Springer-Verlag, New York-Berlin, 1978. MR516508 12.13 .2

[39] Robert E. Williamson Jr., Cobordism of combinatorial manifolds, Ann. of Math. (2) 83 (1966), 1-33. MR0184242 瞇 